\title{
Specialized medial prefrontal-amygdala coordination in other-regarding decision preference
}

\author{
Olga Dal Monte ${ }^{1,2}$, Cheng C. J. Chu', Nicholas A. Fagan' and Steve W. C. Chang ${ }^{1,3,4 凶}$
}

\begin{abstract}
Social behaviors recruit multiple cognitive operations that require interactions between cortical and subcortical brain regions. Interareal synchrony may facilitate such interactions between cortical and subcortical neural populations. However, it remains unknown how neurons from different nodes in the social brain network interact during social decision-making. Here we investigated oscillatory neuronal interactions between the basolateral amygdala and the rostral anterior cingulate gyrus of the medial prefrontal cortex while monkeys expressed context-dependent positive or negative other-regarding preference (ORP), whereby decisions affected the reward received by another monkey. Synchronization between the two nodes was enhanced for a positive ORP but suppressed for a negative ORP. These interactions occurred in beta and gamma frequency bands depending on the area contributing the spikes, exhibited a specific directionality of information flow associated with a positive ORP and could be used to decode social decisions. These findings suggest that specialized coordination in the medial prefrontal-amygdala network underlies social-decision preferences.
\end{abstract}

A ltruistic behaviors and mutually beneficial social exchanges facilitate cohesion among members of a group and help attain collective rewards. While selfish behaviors can be detrimental to these causes, they may be strategically necessary to secure limited resources or to achieve a certain social status. The cognitive operations central to making such social decisions are theorized to recruit multiple brain regions that are sensitive to primary and more abstract rewards, and span cortical and subcortical areas with divergent functional specifications $s^{1-5}$.

Single-neuron studies using social-interaction paradigms have begun to characterize neuronal correlates of social-decision variables concerning conspecific animals in several brain regions. These areas include the anterior cingulate cortex (ACC $)^{6,7}$, the dorsomedial prefrontal cortex ${ }^{8}$, the basolateral amygdala (BLA) ${ }^{9-}$ ${ }^{11}$, the orbitofrontal cortex ${ }^{6,12}$, the striatum ${ }^{13}$ and the lateral prefrontal cortex ${ }^{14,15}$. Of these, the gyrus of the rostral ACC (ACCg) of the medial prefrontal cortex is thought to be particularly specialized in signaling rewarding and motivational information about social partners in humans and monkeys ${ }^{1,16}$. When monkeys express ORPs by choosing to deliver juice rewards to a conspecific monkey over discarding the rewards, some ACCg cells exclusively encode the rewards of the conspecific animal while other cells encode one's own and conspecific's rewards in an indistinguishable manner ${ }^{6}$. By contrast, orbitofrontal cortex or ACC sulcus neurons in this paradigm predominantly signal self-referenced decision variables by modulating firing rates only in relation to one's received or foregone rewards ${ }^{6}$. These findings lend support for the role of the rostral ACCg in computing other-referenced variables $^{16}$. Conversely, BLA neurons exhibit correlated valuetuned activity for encoding choices that result in juice rewards to either themselves or a conspecific monkey ${ }^{9}$, which suggests that BLA neurons utilize a shared metric for decision variables between self and other. Similar characteristics have subsequently been observed in ACCg and BLA neurons in the human brain from an intracranial study ${ }^{17}$.
Coherence between specific nodes in the social brain network likely plays a key role in social behavior. Human functional neuroimaging studies have implicated the importance of correlated activations involving different brain regions in social cognition ${ }^{18,19}$. In prairie voles, frequency-specific coupling between the medial prefrontal cortex and the nucleus accumbens mediates social bonding $^{20}$. Moreover, BLA-projecting ACC neurons are necessary for observational fear-learning in mice ${ }^{21}$. In turn, dysregulated subcortical-medial prefrontal synchrony can result in abnormal social behaviors ${ }^{22}$. However, the neuronal mechanisms underlying interareal synchrony associated with complex social behaviors in primates, such as those related to positive or negative ORPs, remain elusive.

Reciprocal and dense projections between the ACCg and the BLA permit the two nodes to efficiently communicate social and affective information ${ }^{23,24}$. However, whether and how the ACCg and the BLA coordinate activity for social decision-making remain unknown. If coordinated interactions between the ACCg and the BLA are involved in the expression of either a positive or negative ORP concerning the welfare of others, one might expect that distinctive coordination patterns exist for two different types of ORPs. Such interactions may be mediated by a dedicated frequency channel with a specific information flow between the ACCg and the BLA that is associated with expressing a particular ORP. To test this, we investigated how single-neuron spiking and local field potential (LFP) activity between the ACCg and the BLA are dynamically coordinated when monkeys expressed a positive or negative ORP toward a conspecific monkey. We used spike-field coherence as our primary measure as it quantifies how spikes from one region are synchronized to oscillatory LFP signals from another region in discrete time and frequency windows, thereby allowing inspections of synchronous coordination of neural activity between brain areas ${ }^{25,26}$.

We found that synchrony between spikes and LFP oscillations between the two nodes differentiated the positive ORP from the negative ORP. Moreover, these synchrony patterns were specific to select frequency bands and time windows and support a

'Department of Psychology, Yale University, New Haven, CT, USA. ${ }^{2}$ Department of Psychology, University of Turin, Torino, Italy. ${ }^{3}$ Department of Neuroscience, Yale University School of Medicine, New Haven, CT, USA. ${ }^{4}$ Kavli Institute for Neuroscience, Yale University School of Medicine, New Haven, CT, USA. 凶e-mail: steve.chang@yale.edu 


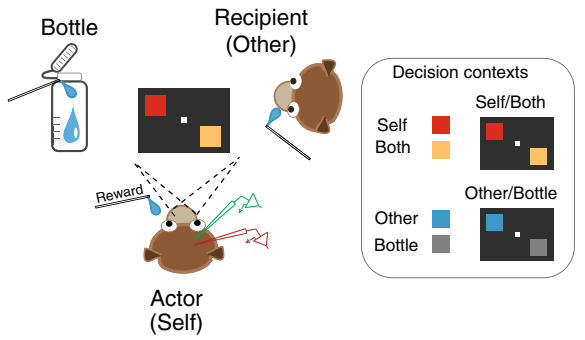

c

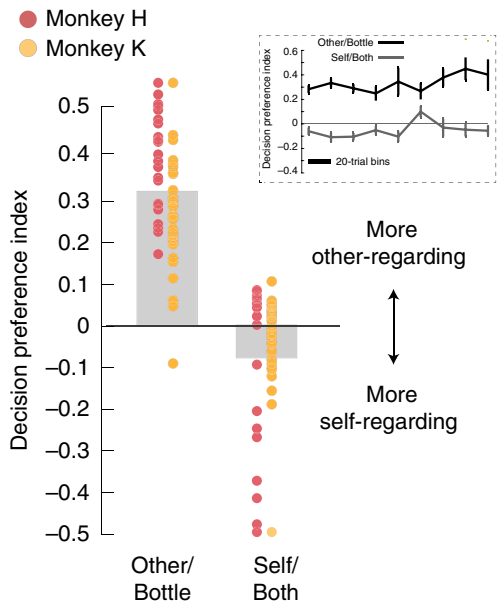

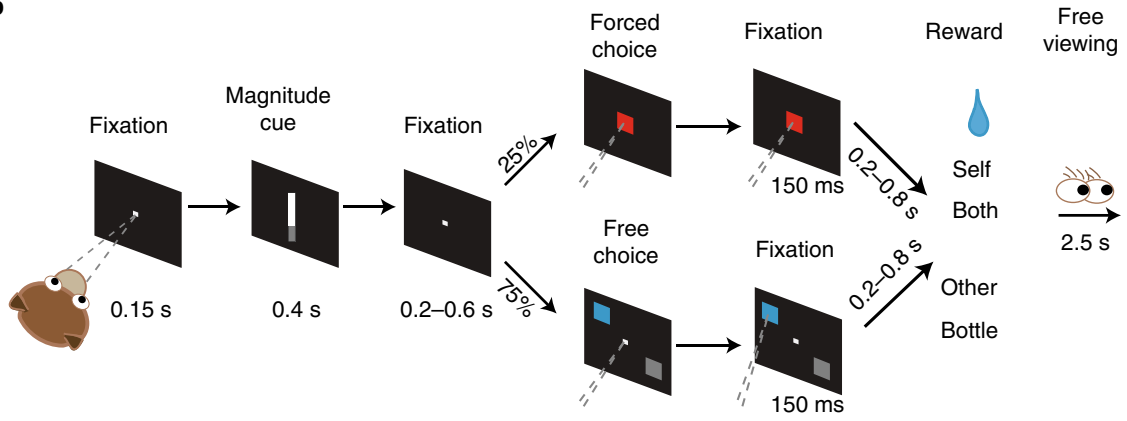

d

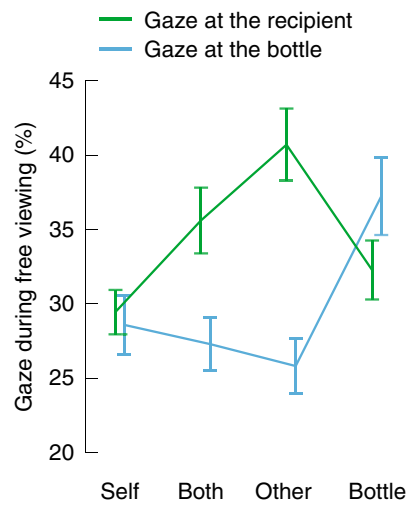

e

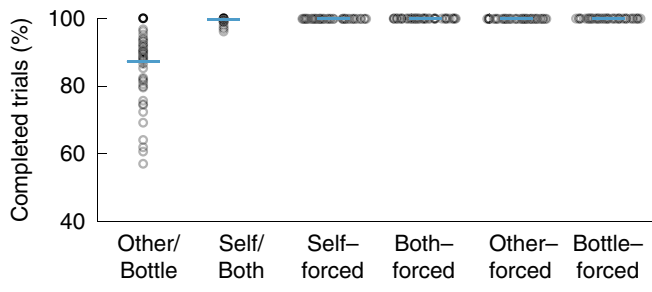

$\mathbf{f}$

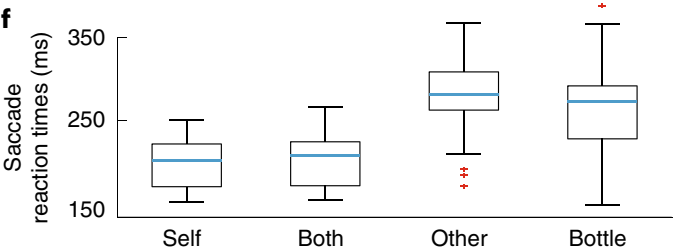

Fig. 1 | Social-reward allocation task and the behaviors associated with social-decision preference. a, Left: experimental set-up involving an actor monkey, a recipient monkey and an operating juice collection bottle. Right: example stimulus-reward outcome mappings for the two distinct contexts for rewarding the actor (Self) or both the actor and the recipient (Both) (Self/Both context), and for rewarding the recipient (Other) or the bottle (Bottle) (Other/Bottle context). b, Task sequence for the social-reward allocation task (see Methods for more detail). c, Monkeys exhibited context-dependent positive and negative ORPs. Decision preferences are expressed as averaged contrast ratios for the two decision contexts. The overlaid data points show the biases for all individual sessions for each subject (mean \pm s.e.m., $n=57$ sessions). The inset shows the preferences over time for each context (mean \pm s.e.m., $n=57$ sessions). d, Social-gaze patterns reflected decisions to deliver juice rewards to the recipient or the bottle as a function of different decisions. Shown are the mean ( \pm s.e.m., $n=57$ sessions) proportions of gaze to the recipient or to the bottle during the free-viewing period for each reward outcome. e, Average proportions of completed free-choice trials for Other/Bottle and Self/Both contexts and completed forced-choice trials for Self, Both, Other or Bottle. Data points show individual sessions $(n=57)$. f, Saccade reaction times (mean \pm s.e.m., $n=57$ sessions) for choosing Self, Both, Other or Bottle. In the boxcar plots, blue lines represent the median, box edges represent the 25th and 75th percentiles, whiskers encompass all non-outlier data points, and outliers are plotted as red crosses.

directional transfer of information between the two nodes. Unique rhythmic coordination of neuronal activity in the primate medial prefrontal-amygdala network may therefore contribute to social decision-making.

\section{Results}

Monkeys exhibit positive and negative ORPs in distinct contexts. Pairs of rhesus macaques (an actor and a recipient) participated in a social-reward allocation task (Fig. 1a,b; Methods). In one decision context (Other/Bottle context), whereby actor monkeys never received juice rewards, actors were free to choose between donating a juice drop to a recipient (Other) and to a juice collection bottle (Bottle). In the other decision context (Self/Both context), whereby actors always received juice rewards, actors were free to choose between delivering rewards to themselves (Self) and to both themselves and the other monkey (Both). This task therefore measures the social-decision preference of the actor without selfreward confounding the choice of one option over the other in two separate contexts.

Actors completed $313 \pm 109$ (mean \pm s.d.) trials per session over 57 sessions (monkey H: $374 \pm 110$ per session, 31 sessions; monkey K: $240 \pm 43$ per session, 26 sessions). Consistent with previous findings using this design ${ }^{6,9,27,28}$, actors preferred to choose Other over Bottle, exhibiting a positive ORP (preference index (mean \pm s.e.m.): $0.32 \pm 0.02, P<0.0001$, Wilcoxon signed-rank) in the Other/Bottle context, but preferred to choose Self over Both, displaying a negative ORP in the Self/Both context $(-0.08 \pm 0.02$, $P<0.001)$ (Fig. 1c). These context-dependent preferences were consistent over time for each session (Self/Both and Other/Bottle context: both $P>0.52$, linear regression) ${ }^{9,27}$ (Fig. 1c). These preferences have also been observed in several different animals in independent studies ${ }^{6,9,27,28}$, are sensitive to pair dominance and familiarity ${ }^{27}$ and are abolished if the recipient monkey is replaced with a juice collection bottle ${ }^{27}$.

Social-gaze patterns differed as a function of decision (Self, Both, Other or Bottle) (two-way ANOVA: $F(3,455)=2.86, P=0.037$ ) and gaze goal (recipient or bottle: $F(1,455)=10.66, P=0.001$ ). Critically, the decision type and gaze goal showed a strong interaction $(F(3,455)=8.75, P<0.0001)$, which indicates that social gaze differed depending on the decision type. Actors overall looked at the recipient $(36 \pm 1 \%($ mean \pm s.e.m. $))$ at a higher rate than to the bottle $(30 \pm 1 \%, P=0.001$, Tukey test). Importantly, after choosing Other, actors looked at the recipient $(41 \pm 2 \%)$ more frequently compared to the bottle $(26 \pm 2 \%, P<0.0001)$. By contrast, actors 
looked at the bottle more often after choosing Bottle $(37 \pm 3 \%)$ than after choosing Other $(26 \pm 2 \%, P=0.002)$ (Fig. 1d). These observations support the notion that actors were acutely aware of the reward outcomes between the conditions in which rewards were either allocated to the recipient or to the bottle; that is, the two outcomes without a self-reward contingency ${ }^{6,9,27,28}$. These contextdependent social-decision preferences provide a behavioral framework for examining the coordination between the ACCg and the BLA in expressing positive and negative ORPs toward a conspecific monkey under different contexts.

For free-choice trials, actors overall completed more Self/Both trials (greater than $99 \%$ for all reward sizes) compared to Other/ Bottle trials ( $87 \%$ for all reward sizes; $F(1,341)=175.12, P<0.0001)$ (Fig. 1e). However, actors were more motivated to complete Other/ Bottle trials when the reward size at stake for either the recipient or the bottle was larger (small: $83 \pm 2 \%$; medium: $87 \pm 2 \%$; large: $90 \pm 2 \% ; F(2,168)=4.3, P=0.02)$. For forced-choice trials, performance reached a ceiling and did not differ between outcomes. Saccade reaction times for free-choice trials differed as a function of decision (Self: $197 \pm 27 \mathrm{~ms}$; Both: $200 \pm 29 \mathrm{~ms}$; Other: $278 \pm 43 \mathrm{~ms}$; Bottle: $271 \pm 59 \mathrm{~ms} ; F(3,215)=59, P<0.0001)$ (Fig. 1f), and was driven by the differences in reaction times for receiving rewards (Self or Both) compared to forgoing rewards (Other or Bottle) $(P<0.0001$, Wilcoxon rank-sum; Self versus Both: $P=0.96$; Other versus Bottle: $P=0.75$; Self or Both versus Other or Bottle: all $P<0.001$, Tukey test).

Coordination of spiking and LFP activity between the ACCg and the BLA. Exploiting context-dependent positive and negative ORPs, we investigated neural coordination for the two types of ORPs between the rostral ACCg (Brodmann areas 24a, 24b and $32)^{29}$ and the $\mathrm{BLA}^{29}$ (Fig. 2). All single units were recorded without any sampling criterion, resulting in 253 ACCg cells and 90 BLA cells. Supplementary Fig. 1 shows basic characterizations of the single-cell activity and example cells with outcome-selective responses. As we have previously characterized single-cell encoding of socialdecision variables within the ACCg and the BLA in the identical task ${ }^{6,9}$, here, we mainly focused on determining the coordination in frequency and time between ACCg and BLA cells.

To determine whether and how neuronal coordination between the BLA and the ACCg might underlie social decision-making, we related the spiking activity of individual cells from each area with LFP oscillations from the other area by calculating spike-field coherence from pairs of neurons and LFP sites ${ }^{25,26}$. Spike-field coherence values were computed from all recorded cells and LFP sites from which we collected neural data without any selection criteria. This resulted in $253 \mathrm{ACCg}$ cells paired with 268 BLA LFP sites $\left(\mathrm{ACCg}_{\text {spike }}-\mathrm{BLA}_{\text {field }}\right)$ and $90 \mathrm{BLA}$ cells paired with $257 \mathrm{ACCg}$ LFP sites $\left(\mathrm{BLA}_{\text {spike }}-\mathrm{ACCg}_{\text {field }}\right)$. In particular, we analyzed coherence during the $150-\mathrm{ms}$ period from the time of acquiring a choice target for the free-choice trials (post-decision epoch) and during the 150 -ms period from the central cue onset for the forced-choice trials to examine coherence patterns specific to active decisions. Importantly, during this epoch, actors were required to maintain gaze fixation on the target for the duration of the epoch to complete their response, thus removing any eye movement confound and allowing us to precisely match the timing and gaze fixation between the free-choice and forced-choice trials. Crucially, coherence values were always compared in a relative, reward-matched fashion (that is, Other-Bottle for positive ORP and Self-Both for negative ORP) such that any observed coherence differences could not be confounded by the contingency of the actor for receiving a juice reward. Thus, actors never received rewards in the Other/Bottle context, but always received rewards in the Self/Both context, and the use of the Other-Bottle and Self-Both contrasts effectively removes any selfreward contingency within the two independent contexts.
Differences in spike-field coherence between the positive ORP (choosing Other over Bottle, Other-Bottle) and the negative ORP (choosing Self over Both, Self-Both) exhibited frequencyspecific coordination as a function of the area that contributed spikes in the pair. Spikes from BLA cells and LFPs from the ACCg $\left(\mathrm{BLA}_{\text {spike }}-\mathrm{ACC}_{\text {field }}\right)$ displayed enhanced coherence in the beta frequency range (defined as $15-25 \mathrm{~Hz}$ ) for the positive ORP $(P<0.0001$, Wilcoxon signed-rank), but suppressed coherence in the same band for the negative ORP $(P<0.0001$; difference between positive and negative ORPs: $P<0.0001$, Wilcoxon signed-rank) (Fig. 3; Supplementary Fig. 2). Figure 3a,c,d, f-k shows spike-field coherence differences between positive and negative ORPs, whereas Fig. 3b,e and Supplementary Fig. 2 separately show spike-field coherence values for each decision preference. This enhanced versus suppressed coherence difference was present immediately before the time of free-choice decision and lasted until around the time of completing the decision (post-decision epoch). In addition, in the gamma frequency range (defined as $45-70 \mathrm{~Hz}$ ), spikes from ACCg cells and LFPs from the BLA $\left(\mathrm{ACCg}_{\text {spike }}-\mathrm{BLA}_{\text {field }}\right)$ exhibited enhanced coherence, again, for the positive ORP $(P<0.0001)$, but suppressed coherence for the negative ORP in the same epoch $(P<0.0001$; difference: $P<0.0001)$ (Fig. $3 \mathrm{~d}-\mathrm{f})$. This coherence difference was also present before the time of free-choice decision and lasted until the time of completing the decision. However, this time course appeared lagged compared with the $\mathrm{BLA}_{\text {spike }}-\mathrm{ACCg}_{\text {field }}$ coherence in the beta band (Fig. 3g; more details below). Moreover, the differences in spike-field coherence between the positive and negative ORPs did not change as a function of the temporal progression within a session for both $\mathrm{BLA}_{\text {spike }}-\mathrm{ACCg}_{\text {field }}$ coherence values (beta band: $P=0.75$; gamma band: $P=0.11$, linear regression) and $\mathrm{ACCg}_{\text {spike }}-\mathrm{BLA}_{\text {field }}$ coherence values (beta band: $P=0.47$; gamma band: $P=0.45$ ).

Next, we investigated whether the observed spike-field coherence was stronger for the subsets of BLA and ACCg cells that significantly differentiated decision outcomes (Self, Both, Other or Bottle; outcome-selective cells) (Supplementary Fig. 1). BLA cells with significant outcome selectivity (37\%) exhibited stronger $\mathrm{BLA}_{\text {spike }}-\mathrm{ACCg}_{\text {field }}$ coherence differences between positive and negative ORPs in the post-decision epoch compared with nonsignificant cells $(P=0.02$, Wilcoxon rank-sum) (Fig. 3 h). By contrast, ACCg cells with significant outcome selectivity (36\%) did not differ in their $\mathrm{ACCg}_{\text {spike }}-\mathrm{BLA}_{\text {field }}$ coherence differences between the two ORPs compared with their nonsignificant counterparts $(P=0.11$; Fig. $3 j)$. These results suggest that outcome-differentiating cells in the BLA may play a more prominent role in $\mathrm{BLA}_{\text {spike }}-\mathrm{ACCg}_{\text {field }}$ synchrony.

Finally, we performed several control analyses to further confirm the enhanced spike-field coupling between the BLA and the ACCg for expressing positive ORPs. We first examined whether the spikefield coherence patterns were influenced by the potential intention of the actor to look in the future at either the face of the conspecific or the bottle during the inter-trial interval, even though the actors were required to maintain steady gaze fixation in the main analysis epoch. Specifically, we tested possible differences in all frequency bands during the post-decision epoch for those trials in which the actors ultimately looked at the face (compared to no future looking) as well as those trials in which they ultimately looked at the bottle (compared to no future looking). For all frequency bands examined, we did not observe marked differences. Crucially, we did not find differences in the beta band $\mathrm{BLA}_{\text {spike }}-\mathrm{ACCg}_{\text {field }}$ coherence $(P=0.98$, Wilcoxon rank sum; Fig. $3 \mathrm{i}$ ) or the gamma band $\mathrm{ACCg}_{\text {spike }}-\mathrm{BLA}_{\text {field }}$ coherence $(P=0.94$; Fig. $3 \mathrm{k})$, which provides support for the idea that the observed spike-field coherence cannot be explained by potential anticipatory attentional allocation to the conspecific or the bottle. Second, we ruled out several additional factors that might explain our findings. The observed spike-field coherence patterns were not simply driven by changes in spiking activity or LFP 


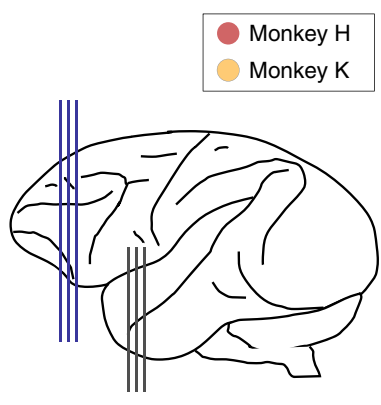

$16 \mathrm{~mm}$
Interaural $37 \mathrm{~mm}$

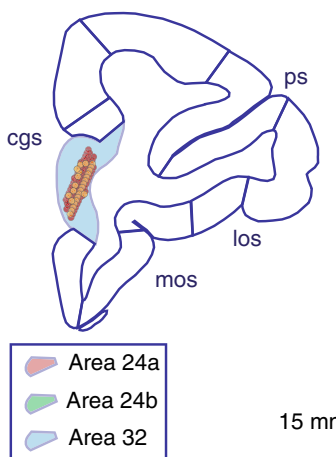

$35 \mathrm{~mm}$

$\mathrm{mm}$
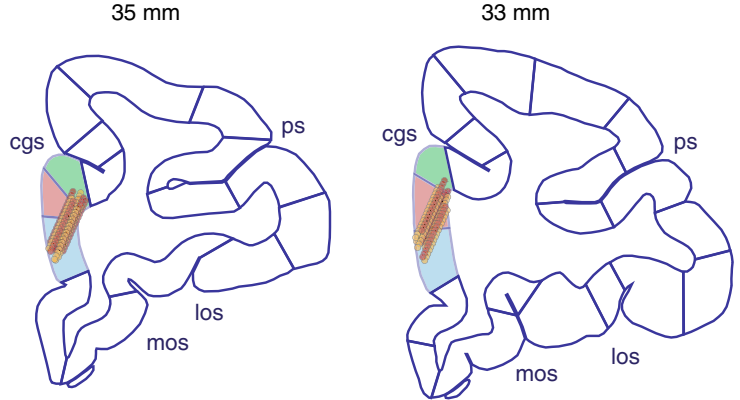
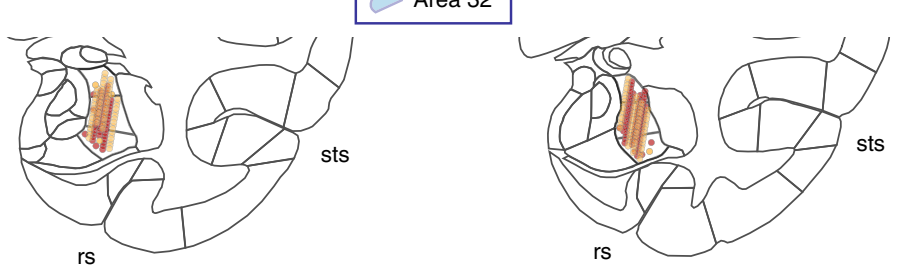

$14 \mathrm{~mm}$

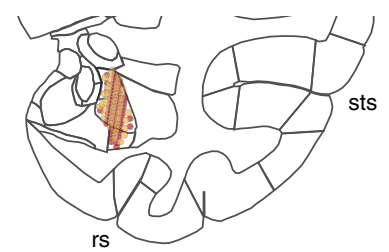

Fig. 2 | Anatomical locations investigated for the coordination of spiking and LFP activity between the BLA and the ACCg. Recording locations for individual cells and LFP sites from monkey $\mathrm{H}$ (red points) and monkey $\mathrm{K}$ (orange points) projected onto standard stereotaxic coordinates of the rhesus macaque brain atlas ${ }^{29}$. For projections for each area, three representative coronal slices were chosen with a 2-mm interaural spacing for the ACCg and a 1-mm interaural spacing for the BLA in the anterior-to-posterior dimension (as shown in the top left cartoon). Selected landmarks are labeled: cingulate sulcus (cgs), principle sulcus (ps), lateral orbitofrontal sulcus (los), medial orbitofrontal sulcus (mos), superior temporal sulcus (sts) and rhinal sulcus (rs). Boxed inset shows region assignments for the ACC Brodmann names based on the Paxinos atlas ${ }^{29}$.

powers (Supplementary Fig. 3; see also Supplementary Fig. 4 for LFP power temporal evolution in the beta and gamma bands) or by a more global-level synchrony or common input signals by comparing them to field-field coherence patterns (Supplementary Fig. 5). When we examined whether the between-region spike-field coherence patterns reported here were different from the within-region spike-field coherence patterns, we found that they were different in several ways (Supplementary Fig. 6). To test whether similar coherence was present even when we constructed positive other-regarding and negative other-regarding choices in different manners ('type 2 contrasts'), we contrasted Both-Self for delivering rewards to the conspecific and Bottle-Other for not delivering rewards to the other monkey. Even with the type 2 contrasts, we found largely consistent spike-field (Supplementary Fig. 7) and field-field coherence patterns (Supplementary Fig. 8), which indicates that the spikefield coherence is not a mere product of a preferred choice, but is driven by positive other-regarding decisions resulting in rewards for others. Finally, we ruled out the possibility that sensory-evoked responses associated with choosing a target stimulus might underlie the differential, frequency-specific coordination. In both beta and gamma frequency bands, the $\mathrm{BLA}_{\text {spike }}-\mathrm{ACCg}_{\text {field }}$ and $\mathrm{ACCg}_{\text {spike }}-$ $\mathrm{BLA}_{\text {field }}$ coherence patterns were not differentially modulated by the onset of a fixation stimulus (Supplementary Fig. 9). Finally, resampling (75\% of randomly selected trials in 1,000 iterations to calculate spike-field coherence values) produced consistent results, which confirms that our results are not driven by outlier cells, sites or trials (positive versus negative ORPs; $\mathrm{BLA}_{\text {spike }}-\mathrm{ACCg}_{\text {field }}$ coherence in the beta band: $P=0.005 ; \mathrm{ACCg}_{\text {spike }}-\mathrm{BLA}_{\text {field }}$ coherence in the gamma band: $P=0.001$; Wilcoxon signed-rank). Taken together, these findings indicate that interareal coherence between the BLA and the ACCg is enhanced for expressing a positive compared with a negative ORP.

Crucially, the coordination of spikes and LFPs observed between the BLA and the ACCg was specific to when the actors made decisions (free-choice). Using pseudo-randomly interleaved forcedchoice trials in which the computer selected the reward outcomes that were otherwise identical, we constructed spike-field coherence differences with matching reward outcomes in the absence of decision-making. We contrasted Other-forced and Bottle-forced trials (forced-choice construct of a positive ORP) for comparing it to a positive ORP, and contrasted Self-forced and Both-forced trials (forced-choice construct of a negative ORP) for comparing it to a negative ORP. The beta $\mathrm{BLA}_{\text {spike }}-\mathrm{ACCg}_{\text {field }}$ coherence and the gamma $\mathrm{ACCg}_{\text {spike }}-\mathrm{BLA}_{\text {field }}$ coherence markedly differed in these comparisons (Fig. 3c,f; Supplementary Fig. 2). The beta $\mathrm{BLA}_{\text {spike }}-\mathrm{ACCg}_{\text {field }}$ coherence $(15-25 \mathrm{~Hz})$, which was selectively enhanced for the positive ORP $(P<0.0001$, Wilcoxon signed-rank), was absent for the forced-choice positive ORP $(P=0.17)$ (difference between free-choice and forced-choice: $P<0.0001$, Wilcoxon rank-sum) (Fig. 3c). Similarly, the gamma $\mathrm{ACCg}_{\text {spike }}-\mathrm{BLA}_{\text {field }}$ coherence $(45-70 \mathrm{~Hz})$, which was again selectively enhanced for the positive ORP $(P<0.0001)$, was absent for the forced-choice positive ORP $(P=0.62$; difference between free-choice and forced-choice: $P<0.0001)$ (Fig. 3f). Therefore, the coordination signatures that differentiated the positive ORP from the negative ORP are unique to making free-choice decisions and not driven by either the visual stimuli or the anticipation of specific reward outcomes.

Given that the beta $\mathrm{BLA}_{\text {spike }}-\mathrm{ACCg}_{\text {field }}$ coherence differences appeared to emerge earlier and terminate sooner than the gamma $\mathrm{ACCg}_{\text {spike }}-\mathrm{BLA}_{\text {field }}$ coherence differences (Fig. 3), we next examined disparities in the coherence onset time to help elucidate any potential functional differences between the two coordination types. The beta $\mathrm{BLA}_{\text {spike }}-\mathrm{ACCg}_{\text {field }}$ coherence began to significantly differentiate positive from negative ORP earlier $(P<0.05$, Wilcoxon signed-rank) than the gamma $\mathrm{ACCg}_{\text {spike }}-\mathrm{BLA}_{\text {field }}$ coherence (Fig. 3g). Moreover, the gamma $\mathrm{ACCg}_{\text {spike }}-\mathrm{BLA}_{\text {field }}$ coherence continued to significantly differentiate positive from negative ORP longer compared with the beta $\mathrm{BLA}_{\text {spike }}-\mathrm{ACCg}_{\text {field }}$ coherence (Fig. $3 \mathrm{~g}$ ). To further investigate temporal profiles, we examined the time at which either spiking or LFP activity began to significantly signal decision outcomes (Supplementary Fig. 10). Spiking activity associated with choosing Other emerged earlier in the BLA compared 
to the ACCg $(P=0.001$, two sample Kolmogorov-Smirnov test). By contrast, there were no such differences for choosing Self, Both or Bottle outcomes among the areas (all $P=0.09$ ) (Supplementary Fig. 10a). Furthermore, we did not observe any temporal differences in LFP power between the two nodes for both the beta (Self, Both, Other and Bottle, all $P>0.38$ ) or the gamma bands (all $P>0.21$ ) (Supplementary Fig. 10b). Finally, we tested whether there were any anatomical differences for the coherence strength. We did not find discernable anatomical gradients for either the beta or gamma spike-field coherence differences between the positive and negative ORPs within ACCg and BLA cells or sites (all comparisons using anterior-posterior, medial-lateral or depth dimension separately, or based on principal component analysis; all $|r|<0.32$, all $P>0.16$, Spearman's correlation).

Directionality of information flow between the ACCg and the BLA for social decisions. Coordination between the ACCg and the BLA may exhibit a specific directionality of information flow that may differ between expressing the two ORPs. We performed a partial directed coherence (PDC) analysis, which is a specialized methodology derived from the Granger principle purposely tailored for analyzing directionality in the frequency-time domain ${ }^{30}$. Without choosing any frequency bands a priori, we observed systematic differences in directional information flow between the ACCg and the BLA as a function of social-decision preference and frequency band. We found a significant influence of the BLA on the ACCg in the beta band (BLA $\rightarrow$ ACCg) for a positive ORP that began around the time of decision-making and continued for the duration of the post-decision epoch (PDC difference between the $\mathrm{BLA} \rightarrow \mathrm{ACCg}$ and the $\mathrm{ACCg} \rightarrow \mathrm{BLA}: P<0.0001$, Wilcoxon signedrank) (Fig. 4a,b). This increase in directional influence occurred in the same frequency range that exhibited an increase in the $\mathrm{BLA}_{\text {spike }}-\mathrm{ACCg}_{\text {field }}$ coherence for the positive ORP. By contrast, we found the opposite pattern for a negative ORP, with a stronger influence of the ACCg on the BLA (PDC difference in the beta band between the ACCg $\rightarrow$ BLA and the BLA $\rightarrow$ ACCg: $P=0.002$ ). Similarly, we found a significant but less pronounced influence of the BLA on the ACCg in the gamma band (BLA $\rightarrow$ ACCg) for the positive ORP (PDC difference in the gamma band between the $\mathrm{BLA} \rightarrow \mathrm{ACCg}$ and the $\mathrm{ACCg} \rightarrow \mathrm{BLA}: P=0.04)$ that appeared later than the BLA $\rightarrow$ ACCg influence in the beta band (Fig. 4c), again with an opposite influence of the ACCg to the BLA for a negative ORP (PDC difference between the ACCg $\rightarrow$ BLA and the BLA $\rightarrow$ ACCg: $P<0.0001)$. However, while we found a frequencydependent BLA $\rightarrow$ ACCg influence for the positive ORP in the beta and gamma bands (compared with the ACCg $\rightarrow$ BLA), the directionality associated with the negative ORP was largely frequency-independent between the BLA $\rightarrow$ ACCg and the ACCg $\rightarrow$ BLA (Fig. 4a,b).

Finally, we observed a similar directionality of information flow in both the BLA $\rightarrow$ ACCg and the ACCg $\rightarrow$ BLA for free-choice compared with forced-choice trials for both ORPs (Supplementary Fig. 11). While we observed a general BLA $\rightarrow \mathrm{ACCg}$ influence on the frequency range that encompassed both the beta and low gamma bands for the positive ORP, the directionality associated with forcedchoice trials was much less frequency-dependent compared with free-choice trials. The directional information flow for the negative ORP showed a strong ACCg $\rightarrow$ BLA influence (again, opposite to the positive ORP) for the negative ORP with a longer time span.

Together, these findings demonstrate the presence of specific information flow directions between the BLA and the ACCg, with a general $\mathrm{BLA} \rightarrow \mathrm{ACC}$ influence for expressing a positive ORP and a $\mathrm{ACCg} \rightarrow \mathrm{BLA}$ influence for expressing a negative ORP. Moreover, although the PDC analyses do not use spikes, the BLA $\rightarrow$ ACCg information flow for the positive ORP was observed in the same beta band that exhibited the enhanced $\mathrm{BLA}_{\text {spike }}-\mathrm{ACCg}_{\text {field }}$ coherence for positive compared with negative ORPs.

Decoding social decisions directly from synchrony between the ACCg and the BLA. To test whether neuronal coordination itself contains decodable information on social decisions, we trained a linear decoder to directly discriminate decisions from spike-field coherence values (Fig. 3). The classifier was trained using randomly selected subsets of $75 \%$ of the trials and later tested on the remaining $25 \%$ of the trials used as inputs, yielding estimates of the decision outcome on each trial.

The first decoder was trained to distinguish between Other and Bottle decisions (positive ORP) from the $\mathrm{BLA}_{\text {spike }}-\mathrm{ACCg}_{\text {field }}$ coherence values in the beta band $(15-25 \mathrm{~Hz})$ or from the $\mathrm{ACCg}_{\text {spike }}-\mathrm{BLA}_{\text {field }}$

Fig. 3 | Spike-field coherence between the ACCg and the BLA shows frequency-specific and free-choice-selective coordination for positive versus negative ORPs. a, Differences in $\mathrm{BLA}_{\text {spike }}-\mathrm{ACC}_{\text {field }}$ coherence values between a positive ORP (Other-Bottle) and a negative ORP (Self-Both) over time, and the frequency aligned to the time of free-choice decision ( $n=403$ pairs). $\mathbf{b}$, Time courses of spike-field coherence values in the beta frequency separately for a positive ORP (light green; Other-Bottle) and a negative ORP (light blue: Self-Both) ( $n=403$ pairs). c, Time courses of beta spike-field coherence differences between a positive ORP and a negative ORP during free-choice trials (purple; $n=403$ pairs) and between the forced-choice construct of a positive ORP (Other-forced - Bottle-forced) and the forced choice construct of negative ORP (Self-forced-Both-forced) for forced-choice trials (gray; $n=287$ pairs). $\mathbf{d}$, Difference in $\mathrm{ACCg}_{\text {spike }}-\mathrm{BLA}_{\text {field }}$ coherence values between a positive ORP and a negative ORP over time and frequency ( $n=1,147$ pairs). Same format as in a. e, Time courses of spike-field coherence values in the gamma frequency separately for a positive ORP (light green) and a negative ORP (light blue; $n=1,147$ pairs). f, Time courses of gamma spike-field coherence differences between positive and negative ORPs for free-choice (purple; $n=1,147$ pairs) trials and between the forced-choice construct of a positive ORP and the forced-choice construct of a negative ORP during forced-choice trials (gray; $n=956$ pairs). $\mathbf{g}$, Average time courses of the beta band BLA $A_{\text {spike }}-A_{C C} g_{\text {field }}$ coherence (red; $n=403$ pairs) and the gamma band $A C C g_{\text {spike }}-$ $\mathrm{BLA}_{\text {field }}$ coherence (blue; $n=1,147$ pairs) differences between the two ORPs (mean \pm s.e.m.). Circles above the lines (in matching colors) show significant differences from zero $(P<0.05$, Wilcoxon signed-rank, two-sided; individual $P$ values are presented in Supplementary Table 1$)$. $\mathbf{h}$, Time courses of the spike-field coherence differences between the two ORPs during free-choice trials in the beta frequency separately for outcome-selective cells (dark pink; $n=171$ pairs) and nonsignificant cells (light pink; $n=232$ pairs). $\mathbf{i}$, Differences in the $B L A_{\text {spike }}-A C C g_{\text {field }}$ coherence values over frequency between when the monkeys ultimately looked (compared with when they did not look) at the face of the conspecific during the inter-trial interval (blue; looking at the conspecific minus no-looking) and when they ultimately looked (compared with when they did not look) at a bottle (gray; future looking at the bottle minus no-looking), collapsed over all outcomes ( $n=403$ pairs). $\mathbf{j}$, Time courses of the gamma band spike-field coherence differences separately for outcome-selective cells (dark pink; $n=548$ pairs) and nonsignificant cells (light pink; $n=599$ pairs). Same format as $\mathbf{h}$. $\mathbf{k}$, Differences in the ACC $g_{\text {spike }}{ }^{-}$ $\mathrm{BLA}_{\text {field }}$ coherence values between looking at the face of the conspecific and the bottle during the inter-trial interval $(n=1,147$ pairs). Same format as $\mathbf{i}$. In $\mathbf{b}, \mathbf{c}, \mathbf{e}, \mathbf{f}$ and $\mathbf{h}-\mathbf{k}$, significant coherence differences from zero (Wilcoxon signed-rank, two-sided) are indicated by asterisks in matching colors, and significant coherence differences between traces (Wilcoxon rank-sum, two-sided) are indicated in black asterisks for the analyzed epoch (gray shading; 0-150 ms) ( ${ }^{\star \star \star} P<0.0001 ;{ }^{\star \star} P<0.001 ;{ }^{\star} P=0.02$; NS, not significant $(\mathbf{c}, P=0.17 ; \mathbf{i}, P=0.98 ; \mathbf{j}, P=0.11 ; \mathbf{f}, P=0.62 ; \mathbf{k}, P=0.94)$ ). In $\mathbf{b}, \mathbf{c}, \mathbf{e}, \mathbf{f}$ and $\mathbf{h}-\mathbf{k}$, the shaded traces represent the s.e.m. centered around the mean. In all plots, the black arrowheads mark the time at which the monkeys completed a freechoice or forced-choice decision by maintaining gaze fixation on a chosen target or cue. 
coherence values in the gamma band $(45-70 \mathrm{~Hz})$. Decoding performance from the beta $\mathrm{BLA}_{\text {spike }}-\mathrm{ACCg}_{\text {field }}$ coherence for discriminating Other from Bottle decisions began to increase before the decision time and peaked around the time of the decision $(P<0.0001$ compared to an empirically derived null distribution, Wilcoxon signed-rank) (Fig. 5a). Conversely, the decoding a
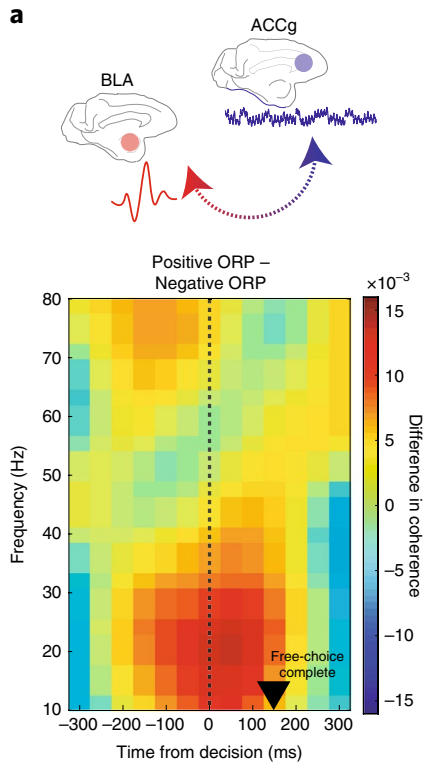

d
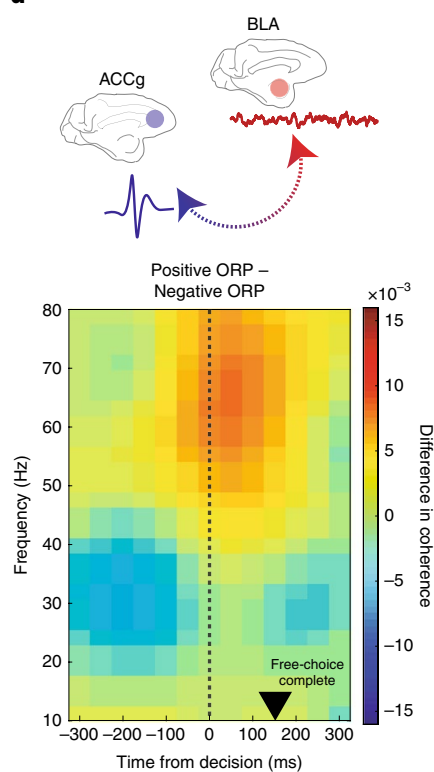

Time from decision $(\mathrm{ms})$ b

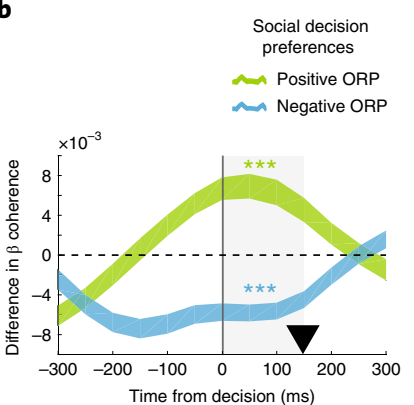

C

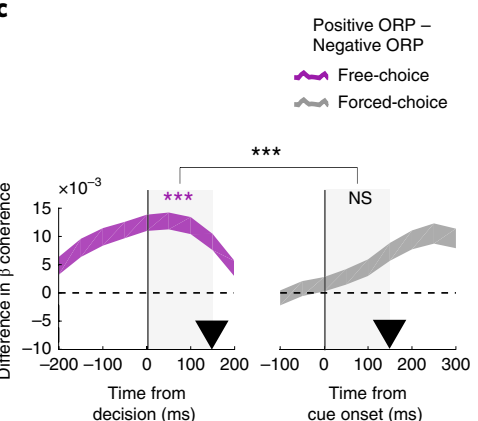

e

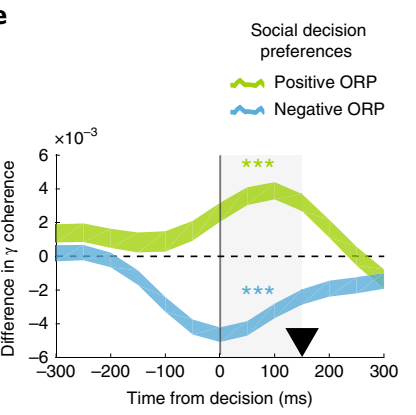

f
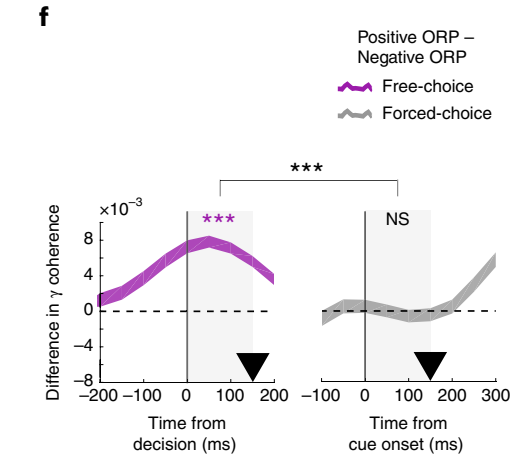

Positive ORP Negative ORP Free-choice $\sim$ Forced-choice
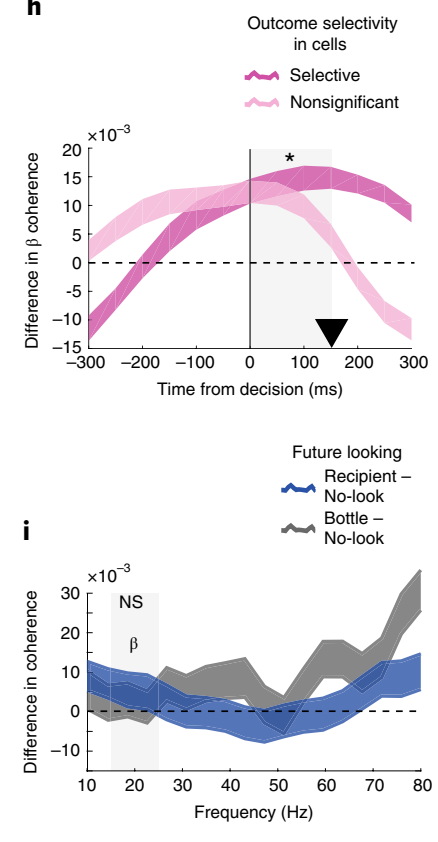

j
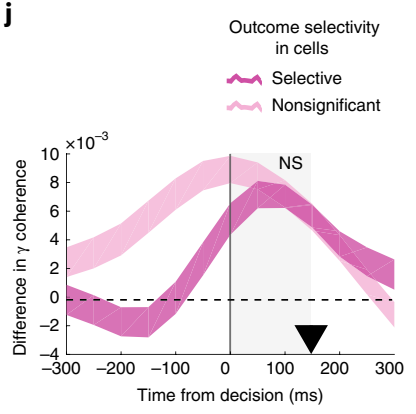

$\mathbf{k}$

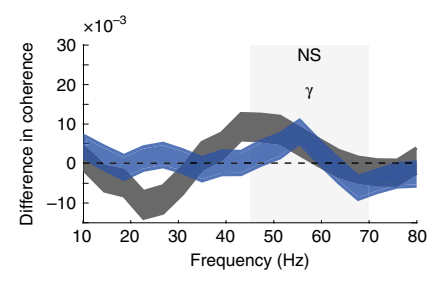

g

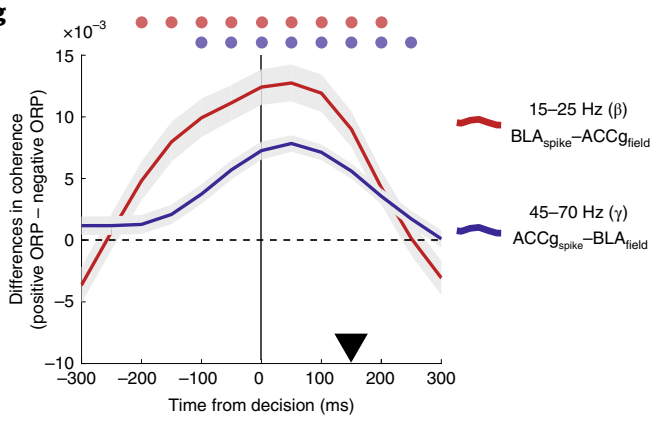


a
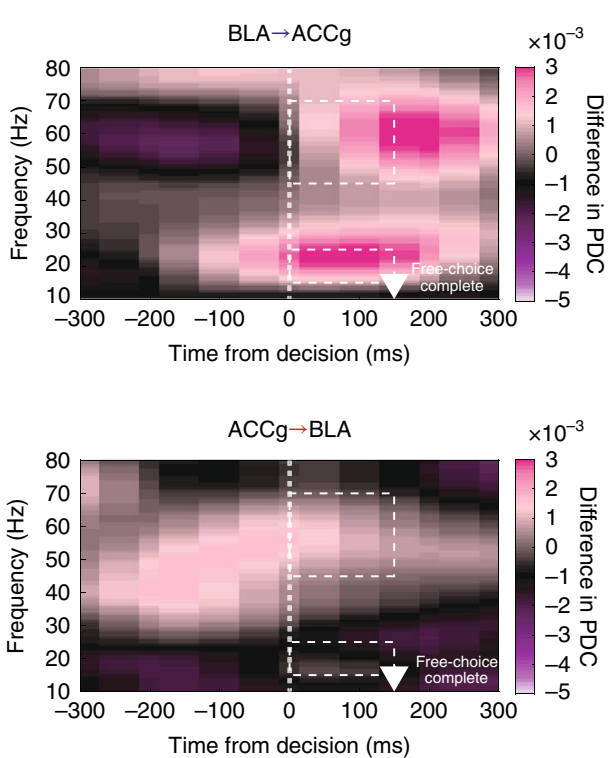

b
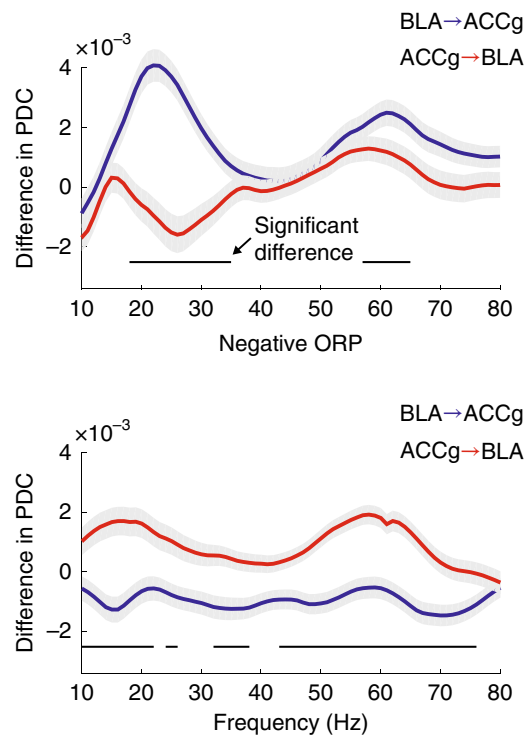

Negative ORP
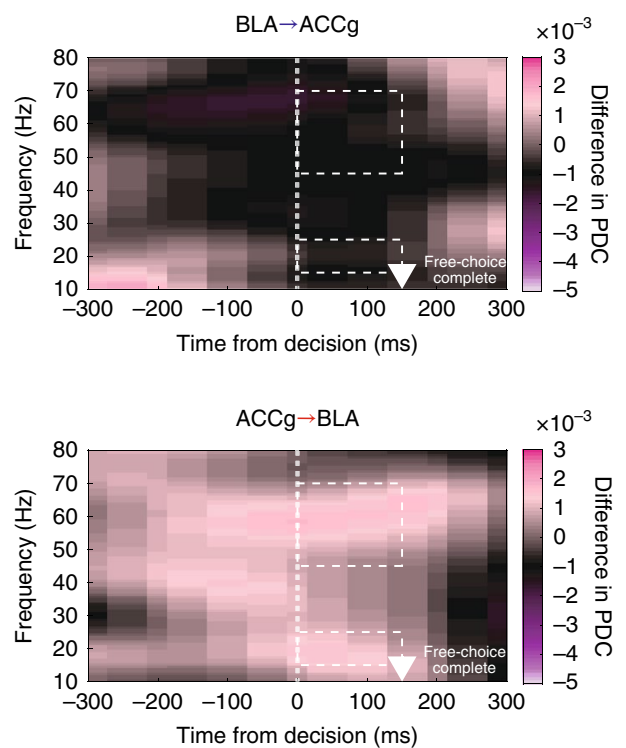

c

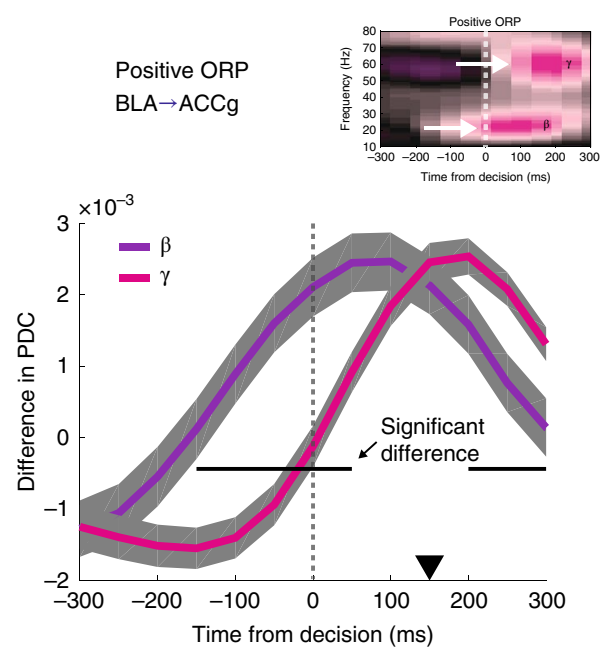

Fig. 4 | Directionality of information flow between the ACCg and the BLA for positive and negative ORPs as a function of time and frequency.

a, Frequency-domain directional influences assessed by PDC for free-choice trials ( $n=593$ pairs). PDC values as a function of time and frequency for the positive ORP (Other-Bottle) for BLA $\rightarrow$ ACCg (top left) and ACCg $\rightarrow B L A$ (bottom left), and PDC values for the negative ORP (Self-Both) for BLA $\rightarrow A C C g$ (top right) and $\mathrm{ACCg} \rightarrow \mathrm{BLA}$ (bottom right). The white arrowheads mark the time at which the monkeys completed a free choice by maintaining fixation on a chosen target for $150 \mathrm{~ms}$. Dotted lines indicate the beta $(15-25 \mathrm{~Hz})$ and gamma $(45-70 \mathrm{~Hz})$ band during the post-decision epoch. b, Quantification of the directionality of information flow during the free-choice decision epoch as a function of frequency for the positive ORP decision (top) and negative $\mathrm{ORP}$ (bottom) for BLA $\rightarrow$ ACCg (blue) and ACCg $\rightarrow B L A($ red). c, Time courses of the beta and gamma band PDC differences for BLA $\rightarrow$ ACCg for the positive ORP. The inset shows the PDC values as a function of time and frequency from which these time course traces were obtained (same as the top left panel in a). In $\mathbf{b}$ and $\mathbf{c}$, horizontal lines indicate significant differences between these PDC values $(P<0.05$, Wilcoxon signed-rank, two-sided; $n=593$ pairs; individual $P$ values are presented in Supplementary Table 1). Shaded regions represent the s.e.m.

accuracy from the gamma $\mathrm{ACCg}_{\text {spike }}-\mathrm{BLA}_{\text {field }}$ coherence for discriminating Other from Bottle decisions was lower at the time of free-choice decision, but gradually improved during post-decision as monkeys fixated on a chosen option to complete the decision (Fig. 5b). The second decoder was trained to distinguish between Self and Both for classifying negative ORPs in the identical frequency bands and times. Compared with the first decoder, the decoding performance was overall lower (positive versus negative ORP in the post-decision epoch: $P<0.0001$ and $P<0.0001$ for decoding from the $\mathrm{BLA}_{\text {spike }}-\mathrm{ACCg}_{\text {field }}$ and $\mathrm{ACCg} \mathrm{g}_{\text {spike }}-\mathrm{BLA}_{\text {field }}$ coherences, respectively) and did not show time-locked increases around the time of free-choice decision, although it was still able to decode above its empirically derived chance level (Fig. 5a,b). To establish whether the improved decoding performance for positive ORPs might emerge earlier for the $\mathrm{BLA}_{\text {spike }}-\mathrm{ACCg}_{\text {field }}$ coherence compared with the $\mathrm{ACCg}_{\text {spike }}-\mathrm{BLA}_{\text {field }}$ coherence, we divided the epoch into the first and second halves and compared decoding between the two ORPs in each phase (beta $\mathrm{BLA}_{\text {spike }}-\mathrm{ACCg}_{\text {field }}$ 
a
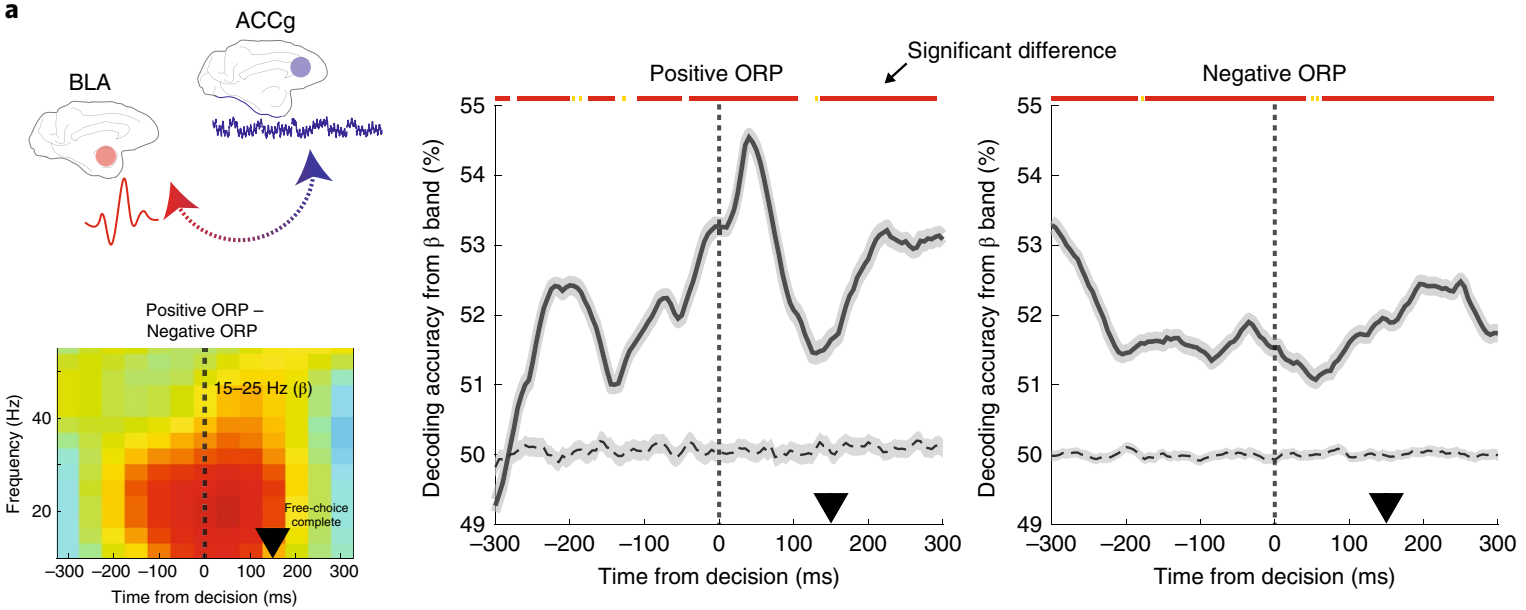

b
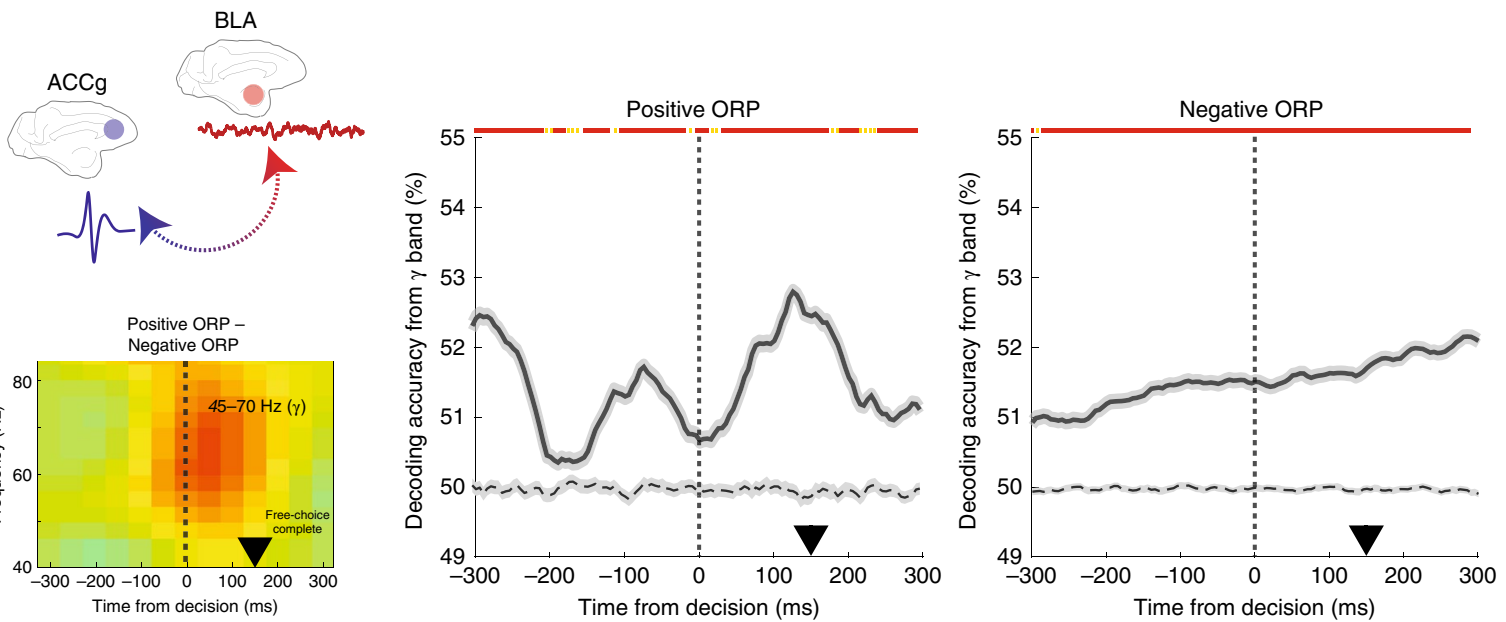

C

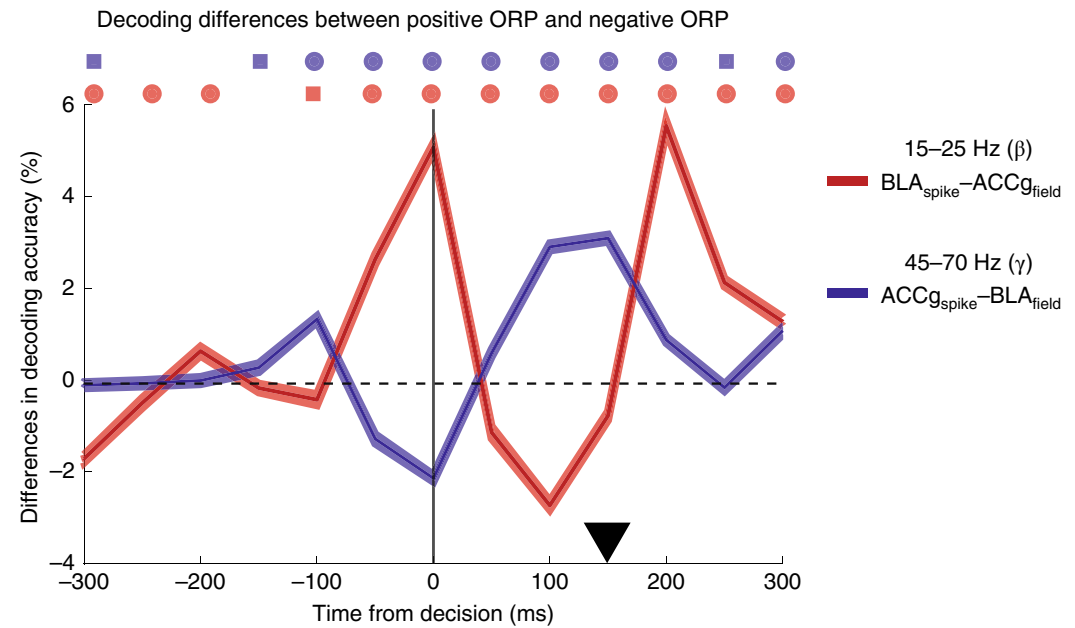

Fig. 5 | Decoding social decisions directly from the spike-field relations between the $A C C g$ and the BLA. a, Decoding performance using the BLA $A_{\text {spike }}-A C C g_{\text {field }}$ coherence differences in the beta band (left inset; $n=403$ pairs; color scale as in Fig. $3 a$ ) for discriminating Other from Bottle (middle) and discriminating Self from Both (right) decisions over time (mean \pm s.e.m.). Lines represent means of 100 permutation iterations with the shaded area representing the s.e.m. Dashed lines represent the empirically determined null distribution. b. Average decoding performance using the $A C C g_{\text {spike }}-B A_{\text {field }}$ coherence in the gamma band (left inset; $n=1,147$ pairs; color scale as in Fig. 3d) for discriminating Other from Bottle (middle) and discriminating Self from Both (right) decisions over time (mean \pm s.e.m.). Lines represent the means of 100 permutation iterations with the shaded area representing the s.e.m.. Same format as in $\mathbf{a}$. In $\mathbf{a}$ and b, colored lines at the top indicate significant differences from the null in each of the 5-ms bin (red: $P<0.0001$, yellow: $P<0.05$, Wilcoxon signed-rank, twosided; individual $P$ values are presented in Supplementary Table 1). c, Differences in decoding performances between Other/Bottle and Self/Both contexts from the beta $B L A_{\text {spike }}-A C C g_{\text {field }}$ coherence (red; $n=403$ pairs) and the gamma $A C C g_{\text {spike }}-B_{\text {field }}$ coherence (blue; $n=1,147$ pairs). Lines represent mean differences from 100 permutation test iterations, with the shaded area representing the s.e.m. Symbols above the lines (in matching colors) show significant differences from zero (circle: $P<0.0001$; square: $P<0.05$, Wilcoxon signed-rank, two-sided; individual $P$ values are presented in Supplementary Table 1). In all plots, the black arrowheads mark the time at which the monkeys completed a free-choice decision by maintaining fixation on a chosen target for $150 \mathrm{~ms}$. 
versus gamma $\left.\mathrm{ACCg} g_{\text {spike }}-\mathrm{BLA}_{\text {field }}\right)$. In the earlier phase, decoding performance was significantly greater for the beta $\mathrm{BLA}_{\text {spike }}-\mathrm{ACCg}_{\text {field }}$ coherence compared with the gamma $\mathrm{ACCg}_{\text {spike }}-\mathrm{BLA}_{\text {field }}$ coherence $(P<0.0001)$, whereas this relationship was reversed in the later phase, such that the relative decoding performance for the gamma $\mathrm{ACCg}_{\text {spike }}-\mathrm{BLA}_{\text {field }}$ coherence was significantly greater than the beta $\mathrm{BLA}_{\text {spike }}-\mathrm{ACCg}_{\text {field }}$ coherence $(P<0.0001)$ (Fig. $\left.5 \mathrm{c}\right)$. These temporal differences in decoding accuracy were consistent with the temporal differences observed between the beta $\mathrm{BLA}_{\text {spike }}-\mathrm{ACCg}_{\text {field }}$ coherence and the gamma $A C C g_{\text {spike }}-\mathrm{BLA}_{\text {field }}$ coherence differences in favor of positive ORPs (Fig. 3g). Although the extent of decoding accuracy for predicting social decisions was low even at the peak level, decoding directly from the synchrony signatures was nevertheless reliable.

\section{Discussion}

Distinct neural populations exhibit synchronized activity that might facilitate information transmission ${ }^{25,31}$. Evidence supports the notion that interareal oscillatory coordination is one of the mechanisms used to regulate a wide range of functions, from visual perception $^{32,33}$, motor planning ${ }^{34}$ and spatial navigation ${ }^{35}$ to higher-order functions underlying working memory ${ }^{36}$, associative learning and decision-making ${ }^{37-40}$. A number of studies have also emphasized the importance of cortical-subcortical interactions ${ }^{20,21,24,38-40}$. Here, we showed that there are specific synchrony signatures of neuronal activity between the BLA and the ACCg in social decision-making.

A positive ORP was associated with enhanced coherence between the spiking of BLA neurons and beta LFP oscillations in the ACCg, and with enhanced coherence between the spiking of ACCg neurons and gamma LFP oscillations in the BLA. By contrast, a negative ORP was associated with suppressed coherence. Thus, enhanced co-engagements of the ACCg and the BLA may promote a positive ORP, whereas co-disengagements may in turn promote a negative ORP. Notably, the coordination exhibited frequency specializations. Frequency-specific coordination may provide separate synchrony 'streams' that might be useful for communicating different information computed locally from each area. Specializations of beta and gamma frequency channels in processing different cognitive information have been observed in the past for cortico-cortical interactions ${ }^{41}$. It has also been suggested that the spiking output of the BLA synchronizes the ACC by influencing oscillations $s^{40}$. Our results suggest that beta frequency may link the presynaptic spiking output of BLA cells with the postsynaptic oscillations at the input of the ACCg to drive interareal synchrony in social decision-making.

Synchrony in the lower frequency range, including beta, is thought to be more robust and tolerant to temporal dynamics of spiking activity due to slower temporal profiles ${ }^{42}$, which perhaps makes lower frequency channels more suitable for synchronizing distant structures compared with higher frequency channels such as gamma. Furthermore, in some cases, beta synchronization can be shortlived, reflecting momentary anticipation, upcoming decisions and internally driving choices in a top-down manner ${ }^{43}$. Synchronization between BLA spikes and ACCg fields may facilitate robust and longrange coordination. Recently, accumulating evidence supports a role of beta synchronization in decision-making, especially when decisions involve context-specific and subjective processes ${ }^{44}$. The increase in beta synchrony between BLA spikes and ACCg fields during postdecisions may therefore signify subjective decision-related feedback associated with positive ORPs. Conversely, synchrony in higher frequencies is likely to be driven by local computations requiring fastspiking GABA-ergic interneurons ${ }^{42,45}$. Gamma frequency has been implicated in generating selective representations of salient stimuli over others ${ }^{46}$. The gamma coherence between ACCg spikes and BLA fields may indicate further local computations in the ACCg following the long-range synchrony initiated by the BLA and may provide support in communicating additional agent-specific computations linked to vicarious reward in the $\mathrm{ACCg}^{6}$.
The directionality of information was largely selective for positive ORPs, with the predominant directional influence from the BLA to the ACCg in the beta frequency greater for positive compared to negative ORPs. This directionality occurred in the same frequency that exhibited enhanced coordination between BLA spikes and ACCg fields for positive ORPs. Crucially, the $\mathrm{BLA}_{\text {spike }}{ }^{-}$ $\mathrm{ACC}_{\text {field }}$ coordination associated with positive ORPs was amplified for the outcome-selective BLA cells. Taking these results together with the earlier emergence of the $\mathrm{BLA}_{\text {spike }}-\mathrm{ACCg}_{\text {field }}$ compared with the $\mathrm{ACCg}_{\text {spike }}-\mathrm{BLA}_{\text {field }}$ coordination, BLA cells that differentiate social-decision outcomes may engage the ACCg for positive ORPs. BLA cells also signal social contextual information, such as socialgaze orientation and facial expressions ${ }^{47,48}$. Future work can test whether and when BLA cells with other known functions transmit information to the rostral ACCg or other medial prefrontal cortical areas to bias social decisions. Furthermore, optogenetic tools would help causally test the function of BLA-ACCg synchrony in social decision-making.

Notably, the BLA-ACCg synchrony was largely specific to active decision-making, compared to trials in which the computer made the decisions, which supports the idea that the interareal synchrony was not simply driven by anticipation of upcoming reward outcomes. Although it is inherently difficult to entirely rule out the possibility that these circuits are less engaged by virtue of not making active decisions, expressing social preference may engage these circuits in unique ways. This hypothesis is supported by two previous observations in the primate BLA that demonstrated specialized codes for computing free-choice, compared with forcedchoice, decisions ${ }^{9,49}$.

In social decision-making, it is imperative to be aware of a chosen option and an ultimate actualization of the reward outcome for either self or other. In the reinforcement-learning theory, post-decision, decision-trace or 'afterstate' signals available during post-decisional monitoring can serve as an important and unique feedback mechanism for efficient learning of actions and reward outcomes ${ }^{50}$. The observed spike-field coherence is unlikely to be directly involved in generating a decision, as the coherence emerged after a stage of formulating a choice for both the beta and gamma bands. Moreover, the increase in the coherence value in both bands specific to positive ORPs only remained until a potential reward could be received, displaying a temporal specificity to the postdecision epoch. We therefore hypothesize that the BLA and the ACCg interact during a post-decision state that is associated with expressing a positive ORP. These synchronous interactions in the prefrontal-amygdala circuits, known for incorporating social, affective and reward information, may occur as a result of synchronized feedback that is useful for adjusting future social decisions. Future work with a specific behavioral design that modulates the fidelity of post-decision monitoring in relation to BLA-ACCg synchrony is necessary to directly test this hypothesis.

Finally, it is worth pointing out some limitations of our work. Although the task had an embedded condition for delivering juice to a non-social entity (Bottle), it remains unknown whether similar coherence would be present during a completely non-social context (despite the fact that the coherence was specific to the reward outcome of the Other, rather than preference per se; Supplementary Fig. 7). Future work should examine how coherence might be differentially modulated between social and non-social contexts. Moreover, although we removed any self-reward contingency within the two independent decision-making contexts (Self-Both from the Self/Both context and Other-Bottle from the Other/Bottle context), it is worthwhile acknowledging that the two contexts were still different, and deriving a positive ORP from the Other/ Bottle context and a negative ORP from the Self/Both context might have influenced our findings. However, the fact that we observed overwhelmingly similar spike-field coherence and field-field 
coherence after deriving a positive ORP from the Self/Both context (Both-Self) and a negative ORP from the Other/Bottle context (Bottle-Other) greatly mitigates this concern.

Overall, our findings support that BLA and ACCg neurons utilize distinct frequency channels and direction-selective coordination in social decision-making. Efficient and strategic coordination occurring between medial prefrontal regions and the amygdala that prioritizes positive over negative ORPs may play an essential role in promoting mutually beneficial social cohesion. In turn, failures in synchronized transmissions along the prefrontal-amygdala network may bias the network to converge toward producing atypical social behaviors.

\section{Online content}

Any methods, additional references, Nature Research reporting summaries, source data, extended data, supplementary information, acknowledgements, peer review information; details of author contributions and competing interests; and statements of data and code availability are available at https://doi.org/10.1038/s41593020-0593-y.

Received: 16 May 2019; Accepted: 17 January 2020; Published online: 24 February 2020

\section{References}

1. Behrens, T. E. J., Hunt, L. T. \& Rushworth, M. F. S. The computation of social behavior. Science 324, 1160-1164 (2009).

2. Bhanji, J. P. \& Delgado, M. R. The social brain and reward: social information processing in the human striatum. Wiley Interdiscip. Rev. Cogn. Sci. 5, 61-73 (2014).

3. Sliwa, J. \& Freiwald, W. A. A dedicated network for social interaction processing in the primate brain. Science 356, 745-749 (2017).

4. Ruff, C. C. \& Fehr, E. The neurobiology of rewards and values in social decision making. Nat. Rev. Neurosci. 15, 549-562 (2014).

5. Seo, H. \& Lee, D. Neural basis of learning and preference during social decision-making. Curr. Opin. Neurobiol. 22, 990-995 (2012).

6. Chang, S. W. C., Gariépy, J.-F. \& Platt, M. L. Neuronal reference frames for social decisions in primate frontal cortex. Nat. Neurosci. 16, 243-250 (2013).

7. Haroush, K. \& Williams, Z. M. Neuronal prediction of opponent's behavior during cooperative social interchange in primates. Cell 160, 1233-1245 (2015)

8. Noritake, A., Ninomiya, T. \& Isoda, M. Social reward monitoring and valuation in the macaque brain. Nat. Neurosci. 21, 1452-1462 (2018).

9. Chang, S. W. C. et al. Neural mechanisms of social decision-making in the primate amygdala. Proc. Natl Acad. Sci. USA 112, 16012-16017 (2015).

10. Grabenhorst, F., Báez-Mendoza, R., Genest, W., Deco, G. \& Schultz, W. Primate amygdala neurons simulate decision processes of social partners. Cell 177, 986-998.e15 (2019).

11. Munuera, J., Rigotti, M. \& Salzman, C. D. Shared neural coding for social hierarchy and reward value in primate amygdala. Nat. Neurosci. 21, 415-423 (2018).

12. Azzi, J. C. B., Sirigu, A. \& Duhamel, J.-R. Modulation of value representation by social context in the primate orbitofrontal cortex. Proc. Natl Acad. Sci. USA 109, 2126-2131 (2012)

13. Baez-Mendoza, R., Harris, C. J. \& Schultz, W. Activity of striatal neurons reflects social action and own reward. Proc. Natl Acad. Sci. USA 110, 16634-16639 (2013)

14. Falcone, R., Brunamonti, E., Ferraina, S. \& Genovesio, A. Neural encoding of self and another agent's goal in the primate prefrontal cortex: human-monkey interactions. Cereb. Cortex 26, 4613-4622 (2016).

15. Nummela, S. U., Jovanovic, V., Mothe, Ldela \& Miller, C. T. Social contextdependent activity in marmoset frontal cortex populations during natural conversations. J. Neurosci. 37, 7036-7047 (2017).

16. Apps, M. A. J., Rushworth, M. F. S. \& Chang, S. W. C. The anterior cingulate gyrus and social cognition: tracking the motivation of others. Neuron $\mathbf{9 0}$, 692-707 (2016).

17. Hill, M. R., Boorman, E. D. \& Fried, I. Observational learning computations in neurons of the human anterior cingulate cortex. Nat. Commun. 7, 12722 (2016)

18. Zaki, J. \& Ochsner, K. The neuroscience of empathy: progress, pitfalls and promise. Nat. Neurosci. 15, 675-680 (2012)

19. Mars, R. B. et al. On the relationship between the "default mode network" and the "social brain". Front. Hum. Neurosci. 6, 189 (2012).

20. Amadei, E. A. et al. Dynamic corticostriatal activity biases social bonding in monogamous female prairie voles. Nature 546, 297-301 (2017).
21. Allsop, S. A. et al. Corticoamygdala transfer of socially derived information gates observational learning. Cell 173, 1329-1342.e18 (2018).

22. Zhan, Y. et al. Deficient neuron-microglia signaling results in impaired functional brain connectivity and social behavior. Nat. Neurosci. 17, 400-406 (2014).

23. Carmichael, S. T. \& Price, J. L. Limbic connections of the orbital and medial prefrontal cortex in macaque monkeys. J. Comp. Neurol. 363, 615-641 (1995).

24. Klavir, O., Genud-Gabai, R. \& Paz, R. Functional connectivity between amygdala and cingulate cortex for adaptive aversive learning. Neuron 80, 1290-1300 (2013).

25. Pesaran, B. et al. Investigating large-scale brain dynamics using field potential recordings: analysis and interpretation. Nat. Neurosci. 21, 903-919 (2018).

26. Fries, P. A mechanism for cognitive dynamics: neuronal communication through neuronal coherence. Trends Cogn. Sci. 9, 474-480 (2005).

27. Chang, S. W. C., Winecoff, A. A. \& Platt, M. L. Vicarious reinforcement in rhesus macaques (Macaca mulatta). Front. Neurosci. 5, 27 (2011).

28. Chang, S. W. C., Barter, J. W., Ebitz, R. B., Watson, K. K. \& Platt, M. L. Inhaled oxytocin amplifies both vicarious reinforcement and self reinforcement in rhesus macaques (Macaca mulatta). Proc. Natl Acad. Sci. USA 109, 959-964 (2012).

29. Paxinos, G., Huang, X.-F. \& Toga, A. W. The Rhesus Monkey Brain in Stereotaxic Coordinates (Academic Press, 1999).

30. Baccalá, L. A. \& Sameshima, K. Partial directed coherence: a new concept in neural structure determination. Biol. Cybern. 84, 463-474 (2001).

31. Buzsáki, G. \& Wang, X.-J. Mechanisms of gamma oscillations. Annu. Rev. Neurosci. 35, 203-225 (2012).

32. Hipp, J. F., Engel, A. K. \& Siegel, M. Oscillatory synchronization in large-scale cortical networks predicts perception. Neuron 69, 387-396 (2011).

33. Womelsdorf, T., Fries, P., Mitra, P. P. \& Desimone, R. Gamma-band synchronization in visual cortex predicts speed of change detection. Nature 439, 733-736 (2006)

34. Wong, Y. T., Fabiszak, M. M., Novikov, Y., Daw, N. D. \& Pesaran, B. Coherent neuronal ensembles are rapidly recruited when making a look-reach decision. Nat. Neurosci. 19, 327-334 (2016).

35. Kahana, M. J., Sekuler, R., Caplan, J. B., Kirschen, M. \& Madsen, J. R. Human theta oscillations exhibit task dependence during virtual maze navigation. Nature 399, 781-784 (1999)

36. Fujisawa, S. \& Buzsáki, G. A 4 Hz oscillation adaptively synchronizes prefrontal, VTA, and hippocampal activities. Neuron 72, 153-165 (2011).

37. Adhikari, A., Topiwala, M. A. \& Gordon, J. A. Synchronized activity between the ventral hippocampus and the medial prefrontal cortex during anxiety. Neuron 65, 257-269 (2010).

38. Antzoulatos, E. G. \& Miller, E. K. Increases in functional connectivity between prefrontal cortex and striatum during category learning. Neuron 83, 216-225 (2014)

39. Brincat, S. L. \& Miller, E. K. Frequency-specific hippocampal-prefrontal interactions during associative learning. Nat. Neurosci. 18, 576-581 (2015).

40. Taub, A. H., Perets, R., Kahana, E. \& Paz, R. Oscillations synchronize amygdala-to-prefrontal primate circuits during aversive learning. Neuron 97, 291-298.e3 (2018)

41. Buschman, T. J. \& Miller, E. K. Top-down versus bottom-up control of attention in the prefrontal and posterior parietal cortices. Science 315, 1860-1862 (2007)

42. Engel, A. K., Fries, P. \& Singer, W. Dynamic predictions: oscillations and synchrony in top-down processing. Nat. Rev. Neurosci. 2 704-716 (2001)

43. Engel, A. K. \& Fries, P. Beta-band oscillations-signalling the status quo? Curr. Opin. Neurobiol. 20, 156-165 (2010).

44. Spitzer, B. \& Haegens, S. Beyond the status quo: a role for beta oscillations in endogenous content (re)activation. eNeuro 4, ENEURO.0170-17.2017 (2017).

45. Cardin, J. A. et al. Driving fast-spiking cells induces gamma rhythm and controls sensory responses. Nature 459, 663-667 (2009).

46. Jia, X. \& Kohn, A. Gamma rhythms in the brain. PLoS Biol. 9, e1001045 (2011).

47. Livneh, U., Resnik, J., Shohat, Y. \& Paz, R. Self-monitoring of social facial expressions in the primate amygdala and cingulate cortex. Proc. Natl Acad. Sci. USA 109, 18956-18961 (2012).

48. Gothard, K. M., Battaglia, F. P., Erickson, C. A., Spitler, K. M. \& Amaral, D. G. Neural responses to facial expression and face identity in the monkey amygdala. J. Neurophysiol. 97, 1671-1683 (2007).

49. Grabenhorst, F., Hernádi, I. \& Schultz, W. Prediction of economic choice by primate amygdala neurons. Proc. Natl Acad. Sci. USA 109, 18950-18955 (2012)

50. Sutton, R. S. \& Barto, A. G. Reinforcement Learning: An Introduction (A Bradford Book, 1998).

Publisher's note Springer Nature remains neutral with regard to jurisdictional claims in published maps and institutional affiliations.

๑ The Author(s), under exclusive licence to Springer Nature America, Inc. 2020 


\section{Methods}

Animals. Two adult male rhesus macaques (Macaca mulatta) were involved in the study as actors (monkeys $\mathrm{K}$ and $\mathrm{H}$; both aged 6 and weighing $7 \mathrm{~kg}$ and $8 \mathrm{~kg}$ ) and two adult female monkeys (aged 6 and 10 years, and weighing $9 \mathrm{~kg}$ and $10 \mathrm{~kg}$ ) were involved only as recipients in the social-reward allocation task. All animals were unrelated and not cage mates. Actors were housed in a colony room with other male macaques, whereas the two female macaques resided in an adjacent colony room with other females. All four subjects were housed in pairs with other animals from the colony, kept on a 12-h light-dark cycle, had unrestricted access to food, but controlled access to fluid during testing. No animals were excluded from our analyses. All procedures were approved by the Yale Institutional Animal Care and Use Committee and complied with the National Institutes of Health Guide for the Care and Use of Laboratory Animals.

Surgery and anatomical localization. All four animals received a surgically implanted headpost (Grey Matter Research) for restraining their head during the experiments. Subsequently, a second surgery was performed on actor monkeys to implant a recording chamber (Crist) to provide access to the ACCg and the BLA. Placement of the chambers was guided by both structural magnetic resonance imaging (MRI, 3T Siemens) scans and stereotaxic coordinates. Before starting the recording experiments, we performed a manganese-enhanced magnetic resonance imaging (MEMRI) session for each actor monkey to precisely localize our recording sites in both the ACCg and the BLA. For MEMRI, we focally infused $2 \mu \mathrm{l}$ of manganese (II) chloride $\left(19.8 \mu{\left.\mathrm{g} \mathrm{l}^{-1}\right)}\right)$ in saline solution in both areas using modified Hamilton syringes that traveled along an identical trajectory to that of the electrodes. We then performed a structural MRI scan $3 \mathrm{~h}$ after the infusion to visualize a bright halo to confirm anatomical locations ${ }^{51}$. All electrophysiological recordings were carried out simultaneously from the ACCg (Brodmann areas 24a, $24 \mathrm{~b}$ and 32$)^{29}$ and the BLA ${ }^{29}$ (Fig. 2).

Social-reward allocation task. Two monkeys (an actor and a recipient) sat in primate chairs (Precision Engineering) $100 \mathrm{~cm}$ from one another at a $90^{\circ}$ angle (Fig. 1a). Each monkey had his own monitor, which displayed identical visual stimuli. Both monkeys had their own juice tubes from which juice drops were delivered via solenoid valves. A third juice tube with its own dedicated solenoid valve delivered juice rewards into an empty bottle (Bottle), which was placed on the opposite side of the recipient (Fig. 1a). To prevent monkeys from forming secondary associations with solenoid clicks, the three solenoid valves were placed in another room and white noise was played in the background during all experimental sessions. An infrared eye-tracking camera (EyeLink 1000, SR Research) continuously recorded the horizontal and vertical eye positions from the actor monkeys.

An actor began a trial by fixating on a central square for $150 \mathrm{~ms}$ with gaze. The reward value at stake for each trial was specified by a magnitude cue displayed as a vertical bar indicating juice volume $(0.2,0.4$ or $0.6 \mathrm{ml})$. The actor was required to maintain gaze fixation on the magnitude cue for $400 \mathrm{~ms}$ Following a variable delay $(200,400$ or $600 \mathrm{~ms})$, the actor was presented with either a free-choice $(75 \%)$ or a forced-choice $(25 \%)$ trial. For free-choice trials, two visual targets appeared at two random peripheral locations on opposite sides of the screen. The actor had $2 \mathrm{~s}$ to make a choice by shifting its gaze to a target and maintaining the fixation on the target for an additional $150 \mathrm{~ms}$ to complete a choice (that is, any break in gaze fixation resulted in an incomplete trial with no further progression in the trial). These choice targets were always presented in two distinct contexts pseudo-randomly presented. In the Self/Both context (50\% of free-choice trials), the actor made decisions to deliver a juice drop to himself (Self) or to both himself and the recipient monkey (Both; the same amount was delivered at the same time to both monkeys). By contrast, in the Other/Bottle context ( $50 \%$ of free-choice trials), the actor made decisions to deliver a juice drop to the recipient monkey (Other) or to the empty juice collection bottle (Bottle). Critically, choices made in the two contexts were reward-matched from the perspective of the actor such that the actor always received a reward in the Self/Both context but never received a reward in the Other/Bottle context. After a variable delay from completing the decision $(200,400,600$ or $800 \mathrm{~ms})$, a juice reward corresponding to the chosen target was delivered to himself (Self), to the recipient (Other), to both monkeys (Both) or to the bottle (Bottle). For forcedchoice trials, only a single central cue was presented on the screen, and the actor had to simply maintain the fixation for $150 \mathrm{~ms}$ to complete the forced-choice decision (that is, any break in fixation resulted in an incomplete trial with no further progression in the trial). These computer-determined reward outcomes occurred with equal frequency, pseudo-randomly ordered. After a variable delay $(200,400,600$ or $800 \mathrm{~ms})$, a juice reward corresponding to the central cue was delivered to himself (Self-forced), to the recipient (Other-forced), to both monkeys (Both-forced) or to the bottle (Bottle-forced). For both free-choice and forced-choice trials, the reward delivery was followed by a 2.5-s inter-tria interval, during which the actor was free to look at the recipient or any other locations in the set-up. A trial was considered incomplete if the actor failed to choose a target or maintain the required 150-ms fixation for free-choice trials or to maintain the required 150 -ms fixation on the cue on forced-choice trials. The incomplete trials were not included in the analyses.
Electrophysiology. LFP and spiking activity were recorded using 16-channel axial array electrodes (U- or V-Probes, Plexon) or single tungsten electrodes (FHC Instruments) placed in each of the recording regions using a 32-channel system (Plexon). At the beginning of each session, a guide tube was used to penetrate the intact dura and to guide electrodes, which were lowered using a motorized multielectrode microdrive system (NaN Instruments) at a speed of $0.02 \mathrm{~mm} \mathrm{~s}^{-1}$. After the electrodes reached the target sites in both the ACCg and the BLA, we waited $30 \mathrm{~min}$ for the tissue to settle before starting each recording session to ensure signal stability. Because some of the data were obtained using two 16-channel electrode arrays, one in the ACCg and the other in the BLA (20\% of the total recording sessions), we randomly assigned 16 uniquely paired LFP sites between the two regions, using a random number generator, to remove redundant inflations of correlation for the relevant data. Otherwise, no cells or LFP sites were excluded from our analyses.

Data analyses. See Data availability and Code availability information at the end of the Online Methods section.

Behavioral analyses. We constructed choice preference indices as contrast $\operatorname{ratios}^{6,9,27,28}$ (equation (1)).

$$
\text { Preference index }=\frac{R_{\mathrm{a}}-R_{\mathrm{b}}}{R_{\mathrm{a}}+R_{\mathrm{b}}}
$$

$R_{\mathrm{a}}$ and $R_{\mathrm{b}}$ were the frequency of particular choices. For the Self/Both context, $R_{\mathrm{a}}$ and $R_{\mathrm{b}}$ were the numbers of Both and Self choices, respectively. For the Other/Bottle context, $R_{\mathrm{a}}$ and $R_{\mathrm{b}}$ were the numbers of Other and Bottle choices, respectively. An index of 1 therefore corresponded to always choosing a positive ORP outcome, -1 corresponded to always choosing a negative ORP outcome and 0 indicated indifference. We also performed regression analysis to quantify changes over time in their behavioral preferences for both Self/Both and Other/Bottle context in each session.

Looking frequency was computed on the basis of the average number of gaze shifts landing on the face of the recipient monkey (the face region of the recipient was empirically mapped and fitted with a rectangle window) or the bottle (mapped empirically with the same-dimensioned window as the face region) during the 2.5 -s inter-trial interval ${ }^{6,9,27,28}$. Decision reaction times, the time from the onset of two targets on free-choice trials to eye movement onset, were computed using a $20^{\circ} \mathrm{s}^{-1}$ velocity criterion ${ }^{6,9,27,28}$.

Spiking and LFP activity. Broadband analog signals were amplified, band-pass filtered $(8-250 \mathrm{kHz})$ and digitized $(40 \mathrm{kHz})$ using a Plexon OmniPlex system. Spiking data were saved for waveform verifications offline and automatically sorted using the algorithm MountainSort ${ }^{52}$. LFP data were analyzed using custom Matlab scripts (MathWorks) and the Chronux signal processing toolbox ${ }^{53}$. Continuous LFP signals from each recording electrode in each area were segmented into 1 -s periods centered on acquiring (that is, saccade offset) the choice target or acquiring the central cue at a sample rate of $1 \mathrm{kHz}$. Raw signals were then band-pass filtered from $2.5 \mathrm{~Hz}$ to $250 \mathrm{~Hz}$. We chose a zero-phase filter to avoid introducing phase distortions to the signals. Signals were normalized by subtracting a reference voltage trace recorded from an independent reference electrode placed in the subdural space to eliminate common noise from each electrode. In a subset of the data where we could accurately compare bipolar and unipolar referencing methods (using 16-channel electrode arrays in both the ACCg and the BLA; $20 \%$ of the total recording sessions), we found similar spike-field coherence results and PDC directional results between the two methods (see ref. ${ }^{54}$ ). Three primary epochs were used to carry out neural data analyses: during the $150-\mathrm{ms}$ window in which the first fixation period was required to begin each trial (baseline epoch); during the 150 -ms period from the time of acquiring (that is, saccade offset) a choice target for free-choice trials (post-decision epoch); and during the 150-ms period after the central cue onset for forced-choice trials (cue epoch). To determine outcome-selective cells from each region, we performed one-way analysis of variance with outcome as the factor (Self, Both, Other or Bottle) using the spiking activity from either the post-decision epoch or reward epoch (50-450 ms from reward onset). Finally, to compare the emergence times of outcomeselective signals in both spiking and LFP activity, we calculated the cumulative distributions of the times at which each cell or LFP site exhibited significant encoding of different outcomes around the time of decision-making relative to the baseline epoch $(P<0.05$, Wilcoxon signed-rank).

Spike-field coherence and field-field coherence. We quantified spike-field coherence levels by examining the phase differences between LFPs and spike signals. We designated one area as the spike contributor and the other area as the field contributor. Spike-field coherence was calculated from two directions: either the ACCg or the BLA as the spike contributor and the other area in the pair as the field contributor. We first binned spikes and LFPs using sliding time-windows of $150 \mathrm{~ms}$, in steps of $50 \mathrm{~ms}$, for a 1-s interval centered on the time of decision for free-choice trials or the cue onset for forced-choice trials. Fourier estimates were then computed by means of a multitaper transformation applied to single trial data; 
we selected a time half-bandwidth product of 2 and multiplied the raw signals by 3 Slepian (orthogonal) tapers ${ }^{55}$. With a $1-\mathrm{kHz}$ sampling rate, this yielded a frequency resolution of $\sim 3.096 \mathrm{~Hz}$. Spectral density estimates were additionally restricted to the $10-80 \mathrm{~Hz}$ interval, considering the Nyquist limit. The spectrum density of point process (spikes) was transformed by applying fast Fourier transform on the discrete data. Coherence was then calculated between two spectrum densities of continuous process (LFP) and point process (spikes) by computing the cross-spectral density of the two processes $\left(x\right.$ and $\left.y ; P_{x y}\right)$ with respect to frequency $(f)$, which was normalized by the product of the power spectral densities of each process $\left(P_{x x}\right.$ and $\left.P_{y y}\right)$ as a function of frequency (equation (2)).

$$
\text { Coherence }=\frac{\left|P_{x y}(f)\right|^{2}}{P_{x x}(f) P_{y y}(f)}
$$

Raw coherence values therefore ranged from 0 to 1 , whereby a perfectly constant phase relationship between the two regions would be indicated by a coherence value of 1 , while an absence of any phase relationship would be indicted by a value of 0 . We contrasted the coherence values between different conditions and obtained an average over pairs of cells and LFP sites, where the spike contributor had at least 1 spike in a $150-\mathrm{ms}$ bin. Linear regression was used to quantify the changes in $\mathrm{BLA}_{\text {spike }}-\mathrm{ACCg}_{\text {field }}$ coherence and $\mathrm{ACCg} \mathrm{g}_{\text {spike }}-\mathrm{BLA}_{\text {field }}$ coherence patterns for both the beta and gamma bands over time within each session.

For calculating within-region spike-field coherence, we used the same approach described above for between-region spike-field coherence, but excluded relating spikes and LFPs originating from the same electrode channels. For looking at the relationships of LFPs between the two regions, field-field coherence was computed using the same format as for the spike-field coherence described above, but with the following exception. Field-field coherence was calculated between two spectrum densities of continuous processes (LFPs from each region) by computing the cross-spectral density of the two processes $\left(x\right.$ and $\left.y ; P_{x y}\right)$ with respect to frequency $(f)$, which was normalized by the product of the power spectral densities of LFP processes from each region $\left(P_{x x}\right.$ and $\left.P_{y y}\right)$ with respect to frequency (same format as in equation (2)).

Directionality of information flow. We calculated the PDC, which is based on the multivariate autoregressive (MVAR) model and is well suited for describing the directionality of information flow between simultaneously recorded time series in the frequency domain ${ }^{30}$. We contrasted the time-varying PDC as (Other) - (Bottle) and (Self) - (Both) for free-choice trials, as well as (Other-forced) - (Bottle-forced) and (Self-forced)-(Both-forced) for forced-choice trials. As for the coherence analyses, we restricted the combinations of pairs to be unique over sites. For example, for the data recorded from a 16-channel array placed in each of the two areas, we randomly selected 16 unique pairs out of $16 \times 16$ pairs to avoid redundancy and undesired inflation in correlations. For each pairwise LFP signals, the parameters of the MVAR model of order $r$ was formulated as follows:

$$
A_{r}=\left[\begin{array}{cc}
a_{i i}^{r} & a_{i j}^{r} \\
a_{j i}^{r} & a_{j j}^{r}
\end{array}\right]
$$

where parameter $a$ reflects a linear relationship between channel $i$ and $j$ at delay $r$. While $r=1 \ldots p$ represents the order of the model. To obtain PDC measures over time, instead of applying an adaptive filtering method to estimate the time-varying autoregressive coefficient, we calculated PDC values based on a sliding window of $150 \mathrm{~ms}$ with a 50 -ms step size, just as we did for the coherence measures. The model order of the MVAR model was estimated by using the post-decision epoch data to minimize Schwarz Bayesian information criteria for all LFP pairs. This resulted in $p=12$, specifying that the current value is predicted by the immediately preceding 12 values in the series. The model extended to the frequency dimension and was defined as follows:

$$
A(f)=I-\left.\sum_{r=1}^{p} A_{r} Z^{-r}\right|_{z=e^{j 2 \pi f}}
$$

where $I$ is the identity matrix and $f$ ranges within 0 to Nyquist frequency. PDC values were then defined by taking the absolute value of $A(f)$ and normalizing by its column vector (see equation $(18)$ in ref. ${ }^{30}$ ). To reduce the covariability of signals between channels due to common sources, we adapted the extended version of classical PDC ${ }^{56}$. The new generalized orthogonalized measure of PDC $\tilde{\psi}$ as a function of time and frequency was defined as follows:

$$
\widetilde{\psi_{i j}}(f)=\frac{1}{\lambda_{k k}^{2}} \frac{\left|\operatorname{Real}\left\{A_{i j}(f)\right\}\right|}{\sqrt{\mathbf{a}_{j}^{H}(f) \Sigma_{w}^{-1} \mathbf{a}_{j}(f)}} \bullet \frac{\left|\operatorname{Imaginary}\left\{A_{i j}(f)\right\}\right|}{\sqrt{\mathbf{a}_{j}^{H}(f) \Sigma_{w}^{-1} \mathbf{a}_{j}(f)}}, \text { if } \mathbf{i} \neq \mathbf{j}
$$

where $\mathbf{a}_{j}$ is the $j$ 's column vector and $A_{i j}$ is the $i j$ th element of $A(f) . H$ denotes the Hamilton transpose of the vector a. $\Sigma_{w}$ is the diagonal covariance matrix from the MVAR noise covariance matrix $w$, where $\lambda_{k k}$ is a diagonal element of $\Sigma_{w}$. For one pair of channels, $(\tilde{\psi})$ was shown in a $2 \times 2$ matrix, where non-diagonal elements represent a directional interaction between channels $i$ and $j$; that is, ACCg $\rightarrow$ BLA or
BLA $\rightarrow$ ACCg. We then calculated and averaged $(\tilde{\psi})$ for all trials in each condition (Self, Both, Other and Bottle) and averaged pairwise sites of PDC for all recording sessions. For testing whether specific frequency bands exhibit significantly different PDC values between conditions for each $\mathrm{ACCg} \rightarrow \mathrm{BLA}$ and $\mathrm{BLA} \rightarrow \mathrm{ACCg}$, we compared PDC values from the same time window used for the main spikefield coherence results.

Linear discriminant analysis. To test the decodability of social decisions directly from spike-field coherence values, we used a standard linear classifier for population decoding ${ }^{57}$. The analysis was run separately for each time-frequency bin (150-ms bin with 5-ms steps) and for each decision context. For a given time-frequency bin and context, the trial-level vector of spike-field coherence values in that bin was extracted, along with the corresponding vector of decision outcomes for each trial. This outcome vector contained Other and Bottle labels or Self and Both labels, depending on the decision context. The decoder was therefore trained to discriminate between binary outcomes on the basis of spike-field coherence values. In the training phase, $75 \%$ of trials were selected at random to train the classifier model. In the testing phase, coherence values for the remaining $25 \%$ of trials were used as inputs, yielding estimates of the decision outcome on each trial.

Decoder performance was assessed as the percentage of test-phase trials that were correctly labeled. The statistical significance of the performance was assessed with a permutation test. For each of the 100 iterations, a null value of the performance of the decoder was obtained by shuffling the decision-outcome labels before training and testing. The analysis therefore produced arrays of matching sizes representing the real and null decoding performance for each (time, frequency, condition and iteration) sequence. Decoding was considered significant if the average performance was higher than the corresponding null performance at least $99 \%$ of the time $(P<0.01$, corrected for false discovery rate for multiple comparisons over frequencies).

General and statistical statements. See the Nature Research Reporting Summary for consistency and transparency in reporting. Data collection and analyses were not performed blinded to the conditions of the experiments. Data collection was randomized for all trial types and stimulus presentations, but had no experimental grouping based on animals. Statistical tests included parametric and nonparametric methods. For both parametric and nonparametric tests, data were well distributed with respect to the assumptions of the test, but this was not formally tested. For tests involving an empirically derived null distribution, the number of iterations and shuffling and resampling procedures were consistent with those of previous publications (for example, see ref. ${ }^{11}$ ). No statistical methods were used to predetermine sample sizes, but our sample sizes (both behavioral and neuronal) were similar to those reported in previous publications (for example, see refs. ${ }^{6,34,41}$ ).

Reporting Summary. Further information on research design is available in the Nature Research Reporting Summary linked to this article.

\section{Data availability}

Behavioral and neural data presented in this paper are available at https://github. $\mathrm{com} /$ changlabneuro/medial-prefrontal-amygdala-coordination-analyses.

\section{Code availability}

Behavioral and neural data analysis codes central to this paper are available at https://github.com/changlabneuro/medial-prefrontal-amygdala-coordinationanalyses.

\section{References}

51. Liu, Y., Yttri, E. A. \& Snyder, L. H. Intention and attention: different functional roles for LIPd and LIPv. Nat. Neurosci. 13, 495-500 (2010).

52. Chung, J. E. et al. A fully automated approach to spike sorting. Neuron $\mathbf{9 5}$, 1381-1394.e6 (2017).

53. Bokil, H., Andrews, P., Kulkarni, J. E., Mehta, S. \& Mitra, P. P. Chronux: a platform for analyzing neural signals. J. Neurosci. Methods 192, 146-151 (2010)

54. Bastos, A. M. \& Schoffelen, J.-M. A tutorial review of functional connectivity analysis methods and their interpretational pitfalls. Front. Syst. Neurosci. 9, 175 (2015).

55. Jarvis, M. R. \& Mitra, P. P. Sampling properties of the spectrum and coherency of sequences of action potentials. Neural Comput. 13, 717-749 (2001)

56. Omidvarnia, A. H. et al. Measuring time-varying information flow in scalp EEG signals: orthogonalized partial directed coherence. IEEE Trans. Biomed. Eng. 61, 680-693 (2013).

57. Saez, A., Rigotti, M., Ostojic, S., Fusi, S. \& Salzman, C. D. Abstract context representations in primate amygdala and prefrontal cortex. Neuron 87, 869-881 (2015). 


\section{Acknowledgements}

We are extremely grateful to B. Pesaran for his guidance on examining oscillatory neural processes throughout the duration of this research. We especially thank D. Lee and A.

Kwan for their thoughtful discussions and suggestions on improving this work. We also thank A. Nair and S. Fan for insightful comments on the manuscript. This work was supported by the National Institute of Mental Health (R01MH110750, R01MH120081, R21MH107853 and R00MH099093), the Alfred P. Sloan Foundation (FG-2015-66028) and the Teresa Seessel Postdoctoral Fellowship.

\section{Author contributions}

S.W.C.C. and O.D.M. designed the study and wrote the paper. O.D.M. performed the experiments. C.C.J.C., N.A.F., O.D.M. and S.W.C.C. analyzed the data.

\section{Competing interests}

The authors declare no competing interests.

\section{Additional information}

Supplementary information is available for this paper at https://doi.org/10.1038/ s41593-020-0593-y.

Correspondence and requests for materials should be addressed to S.W.C.C.

Peer review information Nature Neuroscience thanks Ziv Williams and the other, anonymous, reviewer(s) for their contribution to the peer review of this work.

Reprints and permissions information is available at www.nature.com/reprints. 


\section{natureresearch}

Corresponding author(s): Steve W. C. Chang

Last updated by author(s): Dec 27, 2019

\section{Reporting Summary}

Nature Research wishes to improve the reproducibility of the work that we publish. This form provides structure for consistency and transparency in reporting. For further information on Nature Research policies, see Authors \& Referees and the Editorial Policy Checklist.

\section{Statistics}

For all statistical analyses, confirm that the following items are present in the figure legend, table legend, main text, or Methods section.

n/a Confirmed

$\bigotimes$ The exact sample size $(n)$ for each experimental group/condition, given as a discrete number and unit of measurement

$\square$ A statement on whether measurements were taken from distinct samples or whether the same sample was measured repeatedly

The statistical test(s) used AND whether they are one- or two-sided

Only common tests should be described solely by name; describe more complex techniques in the Methods section.

$\square$ \ A description of all covariates tested

$\square \bigotimes$ A description of any assumptions or corrections, such as tests of normality and adjustment for multiple comparisons

A full description of the statistical parameters including central tendency (e.g. means) or other basic estimates (e.g. regression coefficient)

$\triangle$ AND variation (e.g. standard deviation) or associated estimates of uncertainty (e.g. confidence intervals)

For null hypothesis testing, the test statistic (e.g. $F, t, r$ ) with confidence intervals, effect sizes, degrees of freedom and $P$ value noted

Give $P$ values as exact values whenever suitable.

Х $\square$ For Bayesian analysis, information on the choice of priors and Markov chain Monte Carlo settings

Х $\square$ For hierarchical and complex designs, identification of the appropriate level for tests and full reporting of outcomes

Х $\square$ Estimates of effect sizes (e.g. Cohen's $d$, Pearson's $r$ ), indicating how they were calculated

\section{Our web collection on statistics for biologists contains articles on many of the points above.}

\section{Software and code}

Policy information about availability of computer code

Data collection PsychToolBox in MATLAB (Mathworks, Inc., r2015b), EyeLinkToolBox in MATLAB (Mathworks, Inc., r2015b), Plexon OmniPlex (Plexon, Inc.)

Data analysis

MATLAB (MathWorks, Inc., r2015b). Behavioral and neural analysis codes will be available through https://github.com/changlabneuro upon acceptance of the manuscript.

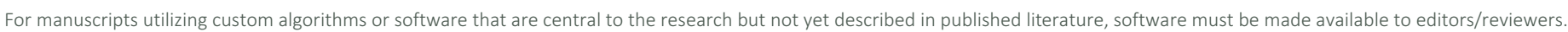
We strongly encourage code deposition in a community repository (e.g. GitHub). See the Nature Research guidelines for submitting code \& software for further information.

Data

Policy information about availability of data

All manuscripts must include a data availability statement. This statement should provide the following information, where applicable:

- Accession codes, unique identifiers, or web links for publicly available datasets

- A list of figures that have associated raw data

- A description of any restrictions on data availability

Behavioral and neural data presented in this paper and the main analysis codes will be available through https://github.com/changlabneuro upon acceptance of the manuscript. 
Please select the one below that is the best fit for your research. If you are not sure, read the appropriate sections before making your selection.
$\bigotimes$ Life sciences
Behavioural \& social sciences
Ecological, evolutionary \& environmental sciences

For a reference copy of the document with all sections, see nature.com/documents/nr-reporting-summary-flat.pdf

\section{Life sciences study design}

All studies must disclose on these points even when the disclosure is negative.

Sample size The study used two actor monkeys and two recipient monkeys ( $n=4$ monkeys). The neural results were obtained from the actor monkeys. This sample size is standard for electrophysiological studies in awake, behaving monkeys. The neural data consisted of $253 \mathrm{ACCg}$ cells paired with 268 BLA LFP sites and 90 BLA cells paired with 257 ACCg LFP sites. This sample size was chosen based on having sufficient amount of data informed by previous studies examining neuronal coherence in the primate brain.

Data exclusions No cells or LFP recording sites were excluded. No selection criterion used during sampling and analyses. The only exception was when we purposefully removed redundant signal correlations from the very same electrodes in multi-channel arrays that could artificially inflate the results (explained in the Methods).

Replication Two monkeys were used for neuronal recording to confirm for replicability of neural signals. Further, as described in the Results section, we confirm our findings by resampling statistics involving subsets of the data.

Randomization Data collection was randomized for all trial types and stimulus presentations but had no experimental grouping based on animals.

Blinding Same researchers trained, collected, and analyzed the data from known animals and blinding was not possible.

\section{Reporting for specific materials, systems and methods}

We require information from authors about some types of materials, experimental systems and methods used in many studies. Here, indicate whether each material, system or method listed is relevant to your study. If you are not sure if a list item applies to your research, read the appropriate section before selecting a response.

\begin{tabular}{l|l}
\multicolumn{2}{c}{ Materials \& experimental sy } \\
\hline $\mathrm{n} / \mathrm{a}$ & Involved in the study \\
$\square$ & $\square$ Antibodies \\
$\square$ & $\square$ Eukaryotic cell lines \\
$\square$ & $\square$ Palaeontology \\
$\square$ & $\square$ Animals and other organisms \\
$\square$ & $\square$ Clinical data
\end{tabular}

Methods

$\mathrm{n} / \mathrm{a}$ Involved in the study

Х $\square$ ChIP-seq

Х Flow cytometry

Х $\square$ MRI-based neuroimaging

\section{Animals and other organisms}

Policy information about studies involving animals; ARRIVE guidelines recommended for reporting animal research

Laboratory animals

Two adult male rhesus macaques (Macaca mulatta) were involved in the study as actors (monkeys $\mathrm{K}$ and $\mathrm{H}$; ages, both 6; weights, 7 and $8 \mathrm{~kg}$ ), and two adult female monkeys (ages, 6 and 10; weights, 9 and $10 \mathrm{~kg}$ ) were involved only as recipients in the social reward allocation task. All animals were unrelated and not cagemates. Actors were housed in a colony room with other male macaques, whereas two female macaques resided in an adjacent colony room with other females. All four subjects were housed in pairs with other animals from the colony, kept on a 12-hr light/dark cycle, had unrestricted access to food, and controlled access to fluid during testing. All procedures were approved by the Yale Institutional Animal Care and Use Committee and in compliance with the National Institutes of Health Guide for the Care and Use of Laboratory Animals.

Wild animals The study did not involve wild animals.

Field-collected samples The study did not involve samples collected from the field.

Ethics oversight

All procedures were approved by the Yale Institutional Animal Care and Use Committee and in compliance with the National Institutes of Health Guide for the Care and Use of Laboratory Animals.

Note that full information on the approval of the study protocol must also be provided in the manuscript. 